\title{
The Importance of Sex Stratification in Autoimmune Disease Biomarker Research: A Systematic Review
}

\author{
Kristy Purnamawati ${ }^{*}$, Jamie Ann-Hui Ong ${ }^{2}$, Siddharth Deshpande ${ }^{2}$, \\ Warren Kok-Yong Tan', Nihar Masurkar'2, Jackson Kwee Low ${ }^{2}$ \\ and Chester Lee Drum ${ }^{3,4,5,6,7}$
}

${ }^{1}$ Biomedical Institute for Global Health Research and Technology (BIGHEART), National University of Singapore (NUS), Singapore, Singapore, ${ }^{2}$ National University of Singapore, Singapore, Singapore, ${ }^{3}$ Cardiovascular Research Institute, National University Health System, Singapore, Singapore, ${ }^{4}$ Department of Medicine, Yong Loo Lin School of Medicine, National University of Singapore, Singapore, Singapore, ${ }^{5}$ Translational Laboratory in Genetic Medicine, Agency for Science, Technology and Research, Singapore, Singapore, ${ }^{6}$ Department of Surgery, Yong Loo Lin School of Medicine, National University of Singapore, Singapore, Singapore, 'Department of Biochemistry, Yong Loo Lin School of Medicine, National University of Singapore, Singapore, Singapore

OPEN ACCESS

Edited by:

J. Michelle Kahlenberg, University of Michigan, United States

Reviewed by: Yun Liang,

University of Michigan,

United States

Pamela McCombe,

The University of Queensland, Australia

*Correspondence: Kristy Purnamawati surkrp@nus.edu.sg

Specialty section: This article was submitted

to Autoimmune and

Autoinflammatory Disorders,

a section of the journal

Frontiers in Immunology

Received: 07 March 2018

Accepted: 15 May 2018

Published: 04 June 2018

Citation:

Purnamawati K, Ong JA-H,

Deshpande S, Tan WK-Y, Masurkar N, Low JK and Drum CL

(2018) The Importance of Sex

Stratification in Autoimmune Disease Biomarker Research: A Systematic

Review.

Front. Immunol. 9:1208. doi: 10.3389/fimmu.2018.01208
The immune system is highly dynamic and regulated by many baseline characteristic factors. As such, significant variability may exist among different patient groups suffering from the same autoimmune disease (AD). However, contemporary research practices tend to take the reductionist aggregate approach: they do not segment $A D$ patients before embarking on biomarker discovery. This approach has been productive: many novel AD biomarkers have recently been discovered. Yet, subsequent validation studies of these biomarkers tend to suffer from a lack of specificity, sensitivity, and reproducibility which hamper their translation for clinical use. To enhance reproducibility in validation studies, an optimal discovery-phase study design is paramount: one which takes into account different parameters affecting the immune system biology. In this systematic review, we highlight need for stratification in one such parameter, i.e., sex stratification. We will first explore sex differences in immune system biology and $A D$ prevalence, followed by reported sex-bias in the clinical phenotypes of two ADs-one which more commonly affects females: systemic lupus erythematosus, and one which more commonly affects males: ankylosing spondylitis. The practice of sex stratification in biomarker research may not only advance the discovery of sex-specific AD biomarkers but more importantly, promote reproducibility in subsequent validation studies, thus easing the translation of these novel biomarkers from bench to bedside to improve AD diagnosis. In addition, such practice will also promote deeper understanding for differential AD pathophysiology in males and females, which will be useful for the development of more effective interventions for each sex type.

\section{Keywords: autoimmune diseases, sex differences, gender, sex stratification, biomarkers}

\section{INTRODUCTION}

Autoimmune diseases (ADs) are a complex class of diseases resulting from the immune system failure to differentiate between self and foreign antigens (1). This misrecognition directs the immune system to attack self-antigens, which consequently modifies the biological functions of the affected tissues. Ultimately, tissue damage and dysfunctions ensue and present as clinical symptoms. However, the onset of clinical symptoms is often delayed and occurs following irreversible damage to the affected tissues 
or organ. There is a global urgency for the discovery of specific and sensitive biomarkers for an early detection of ADs. Additionally, the ideal AD biomarker(s) should also be surrogate for disease severity, progression to disability and response to therapy (2).

The discovery of such biomarkers is not straightforward. Currently, validated biomarkers do not yet fulfill this tall order (we list validated biomarkers from select ADs in Table S1 in Supplementary Material). The immune system biology is dynamic - it varies with genetic background, age, sex, and the environment (3-11). Thus, patients with varying characteristics may present with different clinical phenotype and biomarkers despite suffering from the same AD. Conventional biomarker research strategy has been reductionist and aggregated: they compare all $\mathrm{AD}$ patients and controls of mixed baseline characteristics. This approach, although suboptimal, has been productive, leading to the discovery of many novel AD biomarkers (2). Yet, validation and clinical translation of such novel biomarkers have proven to be challenging, possibly due to: (1) lack of control and patient group stratification and matching; (2) inappropriate biomarker validation strategy; and/or (3) techniques used in clinical trials $(12,13)$. In this review, we highlight the importance of sex stratification in $\mathrm{AD}$ biomarker research prior to the discovery-phase, by drawing attention to the fact that: (1) ADs display stronger female bias and (2) present with different disease trajectories in males and females. There are apparent sex differences in AD pathophysiology. These need to be recognized and hopefully over time, incorporated into $\mathrm{AD}$ research efforts, clinical diagnosis, and management for a better patient outcome.

\section{METHODS}

We assembled a comprehensive list of disease prevalence and associated biomarkers in females and males from different geographical locations. Extensive literature review for the study variables of interest for each of the diseases was mainly carried out using PubMed, although some books and online resources were also consulted. Standard search strategies were used, including medical subject headings such as "disease name" and "biomarker," or "disease name" and other parameters of interest (e.g., "prevalence," "in Japan," "diagnosis," "autoantibodies," etc.). Of note, we searched in futility for "sex/gender differences," "disease name," and "biomarkers," perhaps highlighting the paucity of such studies. In total, we reviewed $>1,000$ abstracts and $>400$ full papers and included papers which fulfilled the following criteria:

1. Table 1, review papers and an immunology textbook describing sex-biased immune responses in humans, i.e., Ref. $(3,4,14)$.

2. Table 2, clinical studies indicating numbers of females and males in their study. Studies in which the male and female numbers were not representative of the disease prevalence and incidence in that particular geography were excluded.

3. Tables 3-6, only reviews [i.e., Ref. (15-19)] or primary clinical research papers reporting at least one significant $(P<0.05)$ sex-bias in clinical phenotype was reported.

4. Table S1 in Supplementary Material, review papers and primary research papers that propose novel serum- or plasma-derived proteomic biomarkers. Only biomarkers that have been validated in multiple clinical studies are included. Often this means seeing multiple papers reporting the same biomarkers. Exclusion criteria include:

a. generic biomarkers such as metabolites and oxidative stress biomarkers, likely to be observed with other diseases;

b. genetic biomarkers such as DNA, RNA, single nucleotide polymorphisms, etc.;

c. biomarkers derived from site-specific fluids, such as synovial and cerebrospinal fluids, urine, tears, fecal matter, etc.

\section{RESULTS}

\section{Males and Females Have Different Biological Landscapes}

A report released by the Institute of Medicine in 2001, "Exploring the Biological Contributions to Human Health: Does Sex Matter?" describes in great detail the different factors that contribute to biological differences in males and females, and how these differences affect health and diseases in these two sexes (11). Beyond the overt differences in reproductive biology, males and females show differences in immune functions, brain organization, pain perception, gene dosing (for genes that escape $\mathrm{X}$-chromosome inactivation) as well as metabolism, lifestyle, and physical performance, all of which may alter pharmacokinetic and pharmaco-dynamic variables in the two sexes (11). Males and females operate from different biological landscapes, be it in healthy, diseased, or recovering states.

To explain autoimmune sex dimorphism, it becomes necessary to first describe the cellular and hormonal interactions found in normal immune regulation and thereafter extrapolate these to autoimmune phenomena. In comparison to the innate immunity, the adaptive immune system is known to be significantly affected by sex. Adult females in general, show stronger immune responses than others, and these responses are partially modulated by sex hormones $(3,4,6,11)$. Other contributing parameters include genetic and environmental factors; we refer the readers to a string of excellent reviews for in-depth discussions of these factors $(3-10)$. We summarize the sex dichotomy in normal immune system biology in Table 1 (3). All components of the innate immunity, such as the toll-like receptor pathway, antigenpresenting cells, dendritic cells, macrophages, granulocytes, and natural killer cells, show stronger activity in females. Despite the lower CD8+ T-cell count, the cytotoxicity of each of these cells is higher in females. As estrogen and progesterone levels wax and wane during the menstrual cycle, the balance between $\mathrm{T}_{\mathrm{H}} 1$ and $\mathrm{T}_{\mathrm{H}} 2$ system fluctuates. This balance and its interactions with other systems such as the $\mathrm{T}_{\mathrm{H}} 17, \mathrm{~T}_{\mathrm{reg}}$, and $\mathrm{B}$-cells dictate the overall immune response. Disruptions to the equilibrium of these different systems lead to different $\mathrm{AD}$ pathologies and disease onsets in males and females (6).

\section{ADs Show Stronger Female Bias}

There are currently more than one hundred identified ADs, with 24 showing high prevalence (occurring in 1 per 10,000 people) 
TABLE 1 | Sex dimorphism of the immune system biology.

\begin{tabular}{|c|c|c|c|c|c|}
\hline \multirow{2}{*}{$\begin{array}{l}\text { Immune } \\
\text { component }\end{array}$} & \multirow[t]{2}{*}{ Cytokines (14) } & \multirow{2}{*}{$\begin{array}{l}\text { Sex differences } \\
\text { (females vs males) (3) }\end{array}$} & \multicolumn{3}{|c|}{ Effects of sex hormones $(3,4,24)$} \\
\hline & & & Estradiol & Progesterone & Androgens \\
\hline \multicolumn{6}{|l|}{ Innate immunity } \\
\hline $\begin{array}{l}\text { Toll-like receptor } \\
\text { (TLR) pathway }\end{array}$ & $\begin{array}{l}\text { Inflammatory cytokines, chemotactic } \\
\text { factors, antimicrobial peptides, type I } \\
\text { interferons (IFN } \alpha \text { and IFN } \beta \text { ) }\end{array}$ & $\begin{array}{l}\uparrow \text { TLR gene expression } \\
\uparrow T L R 7 \text { expression } \\
\downarrow \text { IL-10 production by } \\
\text { TLR-9 stimulated PBMCs }\end{array}$ & $\uparrow$ TLR4, TLR7, TLR9 & $\downarrow$ TLR3, TLR7 & $\downarrow$ TLR4 \\
\hline $\begin{array}{l}\text { Antigen-presenting } \\
\text { cells (APCs) }\end{array}$ & Interleukins: IL 12, IL 17 (20) & $\uparrow$ APC efficiency & $\downarrow$ Antigen presentation (21) & $\begin{array}{l}\uparrow \text { Antigen } \\
\text { presentation (22) }\end{array}$ & $\begin{array}{l}\downarrow \text { Antigen } \\
\text { presentation } \\
\text { (23) }\end{array}$ \\
\hline $\begin{array}{l}\text { Dendritic cells } \\
\text { (DCs) }\end{array}$ & $\begin{array}{l}\text { Interferons: IFN } \alpha \\
\text { Interleukins: IL10, IL12, IL23, IL27, } \\
\text { IL28, IL29, IL37 }\end{array}$ & $\begin{array}{l}\uparrow \text { TLR7 activity } \\
\uparrow \text { Type I interferon } \\
\text { (IFN) activity }\end{array}$ & $\begin{array}{l}\uparrow \text { Activation, TLR7, TLR9, } \\
\uparrow \text { Production of CCL2, IL6, IL8 } \\
\text { and IL12 } \\
\uparrow \text { Expansion of IFN } \gamma \text {-producing } \\
\text { killer DCs from mature splenic DCs } \\
\downarrow \text { CXCL10, IFN } \alpha\end{array}$ & $\begin{array}{l}\uparrow \text { IL10, IL18, CD11C } \\
\downarrow \text { CD40, CD80, } \\
\text { CD86 }\end{array}$ & Not defined \\
\hline Macrophage & $\begin{array}{l}\text { Colony stimulating factors: GM-CSF } \\
\text { Interferons: IFN } \alpha \text {, } \\
\text { Interleukins: IL1 } \alpha, \text { IL } 1 \beta, \text { IL6, IL10, } \\
\text { IL12, IL15, IL18, IL23, IL27, } \\
\text { oncostatin } \\
\text { TNF family: TNF } \alpha\end{array}$ & $\begin{array}{l}\uparrow \text { Activation levels } \\
\uparrow \text { Phagocytic capacity } \\
\uparrow \text { IL-10 production } \\
\downarrow \text { Pro-inflammatory } \\
\text { cytokine production } \\
\downarrow \text { TLR4 expression }\end{array}$ & $\begin{array}{l}\uparrow \text { TLR4 } \\
\downarrow \text { IL } 1 \beta, \text { IL6 and TNF production }\end{array}$ & $\begin{array}{l}\uparrow \mathrm{FIZZ1}, \mathrm{YM} 1 \\
\downarrow \mathrm{iNOS}, \mathrm{NO}\end{array}$ & $\begin{array}{l}\downarrow \mathrm{TNF} \text { and } \\
\text { iNOS/NO }\end{array}$ \\
\hline Eosinophil & IFN $\alpha$, IL16 & & $\downarrow$ Count and mobilization & $\uparrow$ Count & Not defined \\
\hline Neutrophil & $\mathrm{IFN} \gamma, \mathrm{IL} 17$ & $\begin{array}{l}\uparrow \text { Phagocytic capacity } \\
\downarrow \text { TLR expression levels }\end{array}$ & $\begin{array}{l}\uparrow \text { Count, anti-inflammatory activity, } \\
\text { elastase release } \\
\downarrow \text { Chemotactic activity }\end{array}$ & Not defined & $\begin{array}{l}\uparrow \text { Count } \\
\downarrow \text { Kinases and } \\
\text { Leukotriene } \\
\text { formation }\end{array}$ \\
\hline NK Cells & $\begin{array}{l}\text { Interferons: IFN } \gamma \\
\text { Interleukins: IL17, IL26, IL32 } \\
\text { TNF family: TNF } \alpha\end{array}$ & $\downarrow$ Cell count & $\begin{array}{l}\uparrow \text { IFN } \gamma \text {, Granzyme B } \\
\downarrow \text { FASL } \\
\downarrow \text { Cytotoxic activity }\end{array}$ & $\begin{array}{l}\uparrow \text { Cell count and } \\
\text { apoptosis (caspase } \\
\text { dependent) }\end{array}$ & Not defined \\
\hline \multicolumn{6}{|l|}{ Adaptive immunity } \\
\hline T-cells & $\begin{array}{l}\text { Colony stimulating factors: GM-CSF, } \\
\text { M-CSF } \\
\text { Interferons: IFN } \gamma \\
\text { TNF family: TNF } \alpha \text {, Iymphotoxin, } \\
\text { CD40L, FasL, CD27L, CD30L, APRIL, } \\
\text { LIGHT, BAFF } \\
\text { Interleukins: IL2, IL4, IL5, IL6, } \\
\text { IL9, IL10, IL13, IL16, IL24, IL32, } \\
\text { oncostatin } \\
\mathrm{T}_{H} 1 \text { cells: IL20 } \\
\mathrm{T}_{H} 2 \text { cells: IL21, IL25, IL31 } \\
\mathrm{T}_{\mathrm{H}} 17 \text { cells: IL17, IL26 } \\
\mathrm{T}_{\text {reg: }} \text { IL35 }\end{array}$ & $\begin{array}{l}\uparrow \text { CD4+ T cell count (25) } \\
\uparrow \text { CD4/CD8 T cell ratio } \\
\uparrow \text { Activated T cells count } \\
\uparrow T \text { cell proliferation } \\
\downarrow \text { CD8+ count } \\
\uparrow \text { CD8+ cytotoxicity } \\
\uparrow T_{H} 2 \text { bias } \\
\downarrow T_{H} 1 \text { bias } \\
\downarrow T_{\text {reg }} \text { cell count } \\
\uparrow T_{H} 1 \text { cytokine secretion }\end{array}$ & $\begin{array}{l}\text { Low estradiol: } \uparrow \text { IFN } \gamma \text { and } T_{H} 1 \text { cells } \\
\text { responses } \\
\text { High estradiol: } \uparrow I L 4 \text { and } T_{H} 2 \text { cells } \\
\text { responses } \\
\uparrow T_{\text {reg }} \text { cell count } \\
\uparrow \text { Response of CD8+ T cells } \\
\uparrow \text { secretion of IFN } \gamma \text { and IL10 (24) } \\
\uparrow \text { CD4+ CCR } 1 \text { and CCR5 } \\
\text { expression } \\
\downarrow T_{H} 17 \text { cell count and IL17 } \\
\text { expression } \\
\downarrow \text { CD4+ TNF production }\end{array}$ & $\begin{array}{l}\downarrow T_{H} 1 \text { cells activity } \\
\uparrow T_{H} 2 \text { cells activity } \\
\downarrow \% \text { of } T_{H} 17 \\
\downarrow \% \text { of } T_{\text {reg }} \\
\downarrow \text { Response of } \\
\text { CD } 8+T \text { cells }\end{array}$ & $\begin{array}{l}\downarrow \text { IFN } \gamma \\
\text { production } \\
\text { by } T_{H} 1 \text { cells } \\
\downarrow T_{H} 2 \\
\text { expression of } \\
\text { IL4, IL5 and } \\
\text { GATA3 } \\
\uparrow \text { IL17 } \\
\uparrow T_{\text {reg cell }} \\
\text { count } \\
\downarrow \text { CD8+ cell } \\
\text { count and } \\
\text { activity }\end{array}$ \\
\hline B-cells & Lymphotoxin & $\begin{array}{l}\uparrow \text { B-cell count } \\
\uparrow \text { Antibody production }\end{array}$ & $\begin{array}{l}\uparrow \operatorname{lgM} \text { and IgG prodction } \\
\uparrow \text { Survival of autoreactive B cells } \\
\downarrow \text { Negative selection of naïve B cells }\end{array}$ & $\begin{array}{l}\uparrow \text { Total antibody } \\
\text { production } \\
\downarrow \text { Autoantibodies }\end{array}$ & $\downarrow$ Response \\
\hline
\end{tabular}

(212). $71 \%$ of these common ADs (Figure 1 below, in bold) are more prevalent in females than males ( $>50 \%$ female prevalence), suggestive of a stronger female bias (213). However, these data were an aggregated one from "world," and "USA" (213). In Table 2, we stratify some of these ADs by geographical location (as a proxy for ethnicity), in order to gain a better insight of each AD's prevalence in different parts of the world. In addition, we include three $\mathrm{ADs}$ which have been reported to be more prevalent in males: idiopathic pulmonary fibrosis (IPF), ankylosing spondylitis (AS), and Guillain-Barré syndrome (GBS).
Table 2 shows that female predominance prevails in all three systemic ADs. Regardless of the geography, females are more than thrice as likely as males to suffer from systemic ADs. For instance, Spanish women are 18.6 times more likely than men to suffer from Sjögren's syndrome (SS). A similar trend is also observed with endocrine ADs: Grave's disease and Hashimoto's thyroiditis (HT). In India, the female to male ratio for HT is an astonishing 21.7 to 1 . Another strong female bias (at least a 2:1 female to male ratio worldwide) is observed with some gastrointestinal and hepatic ADs (primary biliary cholangitis 
TABLE 2 | Female-to-male ratio of autoimmune diseases (ADs) from different regions of the world.

\section{Class}

Disease

Female: Male ratio

Scandinavia Europe

\section{Australia or New}

Zealand

Systemic and connective tissue

Systemic lupus erythematosus

$6(26)-11.6(27): 1$

4.7:1 (28) (Sweden)

5.8:1 (29) (France)

$4.4: 1(30)$

7.8 (31)-11.4 (32):1 (China) 6:1 (33) (Korea) 8.2:1 (34) (Japan)

Sjögren's syndrome $5.5: 1(35)$

8 (28)-8.7 (36):1 (Sweden)

$15.8: 1$ (37)

18.6:1 (38) (Spain)

8.3:1 (39)

4.2:1 (40) (India)

17.4:1 (41) (Japan) 17:1 (42) (China)

Pulmonary

Idiopathic pulmonary fibrosis

$0.9(43)-1.2(44): 1$

0.3:1 (45) (Denmark)

0.4:1 (46) (Sweden)

$0.7: 1$ (47) (Spain)

0.6:1 (48) (UK)

$0.5: 1(50)$

0.3:1 (51) (Korea)

0.3:1 (49) (Germany)

Skin

Scleroderma (also systemic)

4.8:1 (53)

3.2:1 (28) (Denmark)

3.8:1 (54) (Norway)

9.7:1 (55) (Italy)

4.7 (56)-7.2:1 (57) (UK)

7.4:1 (59)

0.4:1 (52) (Japan)

10.4:1 (58) (France)

\begin{tabular}{|c|c|c|c|c|c|}
\hline Psoriasis & $1(62)-1.3(63): 1$ & $\begin{array}{c}1.1: 1 \text { (28) (Denmark) } \\
1: 1 \text { (64) (Norway) }\end{array}$ & $\begin{array}{c}\text { 1.3:1 (65) (Spain) } \\
0.8: 1 \text { (66) (Germany) } \\
1: 1 \text { (67) (UK) }\end{array}$ & $2: 1(68)$ & 0.6:1 (69) (Japan) \\
\hline
\end{tabular}

\section{Hematopoetic and vascular}

Antiphospholipid syndrome

$3.6: 1(70,71)$

4.5:1 (72) (Norway)

2.1:1 (73) (Spain)

$1.7: 1$ (74) (Italy)

10:1 (75) (UK)

Immune thrombocytopenic

$1.1(78)-1.4(79,80): 1$

1.7:1 (81) (Denmark)

1.7:1 (82) (France)

1.4:1 (83) (Germany)

1.3:1 (84) (UK)

$1.3(85)-1.6(86$,

$87): 1$

3.5:1 (94) (Greece)

4.4:1 (95) (France)

Grave's disease

$6: 1(92)$

5.8:1 (93) (Denmark)

5.4:1 (94) (Greece)

4.4:1 (28)

$0.8: 1(103)$

$11.8: 1(98)$

$1: 1(102)$

Type 1 diabetes (adult*)

0.9-1.5:1 (105, 106)
.

Gastrointestinal and liver

Primary biliary cholangitis

10 (109)-12.4 (110):1

4.1:1 (28)

$4.1: 1(116)$

Autoimmune hepatitis

$3.17: 1$ (117) (Sweden)

4:1 (118) (Norway)

2.6:1 (119) (Denmark)

0.9:1 (129)

$1: 1(72)$

$1.1: 1(28)$

$1: 1(129)$

Crohn's disease

$1.3(74)-2.7(75): 1$

1.8:1 (28) (Denmark)

1.2:1 (130) (Finland)

2.4:1 (131) (Sweden)

Musculoskeletal

Ankylosing spondylitis

Celiac disease (adulta) (CoD)

$0.3: 1(135)$
0.5:1 (28) (Denmark)

$0.4: 1$ (136) (Finland)

0.5:1 (137) (Sweden)
5.4:1 (76) (Japan)

4.4:1 (77) (Singapore)

$1.6(88)-2.2(89): 1$ (China)

1.9:1 (90) (India)

4.3:1 (91) (Singapore)

.5-2.8:1 (97) (China)

10.7:1 (100) (China)

21.7:1 (101) (India)

1.3:1 (107) (India)

1.4:1 (108) (Japan) $0.6: 1$ (104) (Spain)

0.5-0.8:1 (104, 105) (UK)

1:1 (105) (Germany)

7.9:1 (111) (France) 12.6:1 (112) (UK)

5.5:1 (120) (Spain)

7.1:1 (121) (Italy)

2.7:1 (122) (Germany)

0.9:1 (70) (France)

0.8:1 (71) (Western EU)

1.32:1 (70) (France)

0.7:1 (71) (Western EU)

1:1 (130) (Germany)

0.6:1 (130) (Italy)

$0.5: 1$ (130) (UK)

0.2:1 (138) (Greece)

0.4:1 (139)
0.3:1 (140) (China)
0.2:1 (141) (India) 0.2-0.3:1 (142) (Japan)

10.5:1 (114) (China) 6.5:1 (115) (Japan)

6.7:1 (125) (Japan)

5.9:1 (126) (China)

8.4:1 (127) (India)

11:1 (128) (Singapore)

1.05:1 (76) (India)

0.7:1 (77) (Asia)

0.9:1 (73) (Japan)

1:1 (76) (India)

0.6:1 (77) (Asia) 0.4:1 (73) (Japan)

0.7:1 (133) (India)

1.3:1 (134) (China)

(Continued) 
TABLE 2 | Continued

\begin{tabular}{|c|c|c|c|c|c|}
\hline \multirow{2}{*}{$\begin{array}{l}\text { Class } \\
\text { Disease }\end{array}$} & \multicolumn{5}{|c|}{ Female: Male ratio } \\
\hline & North Americas & Scandinavia & Europe & $\begin{array}{c}\text { Australia or New } \\
\text { Zealand }\end{array}$ & Asia \\
\hline Rheumatoid arthritis & $2.6: 1(143)$ & 2.2:1 (28) (Denmark) & $2.2: 1(144)(\mathrm{UK})$ & & 3.8:1 (140) (China) \\
\hline Psoriatic arthritis & $0.7: 1(145)$ & $\begin{array}{l}1.23: 1 \text { (137) (Sweden) } \\
0.6: 1 \text { (146) (Norway) }\end{array}$ & $0.2: 1$ (138) (Greece) & & \\
\hline \multicolumn{6}{|l|}{ Neurological } \\
\hline Multiple sclerosis & $2.6: 1(147)$ & 2 (148)-2.3 (28):1 (Denmark) & 2.4:1 (149) (France) & $2.3(150)-4.5(151): 1$ & $\begin{array}{c}\text { 1.8:1 (152) (China) } \\
2.9: 1 \text { (153) (Japan) } \\
\text { 1.7:1 (154) (India) }\end{array}$ \\
\hline Myasthenia gravis & $1.4: 1(155)$ & $1.1: 1(28)$ & $\begin{array}{c}\text { 1.9:1 (156) (Italy) } \\
1.4: 1 \text { (157) (France) }\end{array}$ & $1.3: 1(158)$ & $\begin{array}{c}\text { 2:1 (159) (Japan) } \\
\text { 0.4:1 (160) (India) } \\
\text { 1.15:1 (161) (China) }\end{array}$ \\
\hline Guillain-Barré syndrome & $0.8: 1(162)$ & $\begin{array}{l}\text { 0.6:1 (163) (Finland) } \\
0.8: 1 \text { (164) (Sweden) }\end{array}$ & $\begin{array}{c}0.6: 1 \text { (165) (Italy) } \\
0.6(166)-0.8(167): 1 \text { (UK) }\end{array}$ & $0.6: 1(168)$ & $\begin{array}{c}0.7: 1 \text { (169) (India) } \\
0.7: 1 \text { (170) (China) } \\
0.6: 1 \text { (171) (Japan) }\end{array}$ \\
\hline
\end{tabular}

Data reflect non-pregnant females.

${ }^{a}$ Adult: $15 \leq$ Age $\leq 65$.

TABLE $\mathbf{3}$ | Sex differences in systemic lupus erythematosus clinical phenotypes.

\begin{tabular}{|c|c|c|c|}
\hline Clinical phenotype & $\begin{array}{l}\text { Studies showing phenotype is } \\
\text { increased in males }\end{array}$ & $\begin{array}{l}\text { Studies showing phenotype is increased in } \\
\text { females }\end{array}$ & $\begin{array}{l}\text { Studies showing } \\
\text { statistically in insignificant } \\
\text { in males and females }\end{array}$ \\
\hline Mortality & $(27,172)$ & & \\
\hline Disease activity & $(17,27,173)$ & & $(174)$ \\
\hline Alopecia & & $(18,27,32,175-182)$ & (183) \\
\hline Photosensitivity & & $(18,27,176,178,179,184,185)$ & $(181,183)$ \\
\hline Discoid lesions & $(32,181,186-188)$ & & (183) \\
\hline Malar rash & & $(18,27,181,186,189)$ & (183) \\
\hline Raynaud's phenomenon (RP) & & $(17,27,32,176-179,184,185,190,191)$ & \\
\hline $\begin{array}{l}\text { Musculoskeletal (myositis, tendonitis, } \\
\text { arthralgia/arthritis) }\end{array}$ & $(178)$ & $\begin{array}{l}(17,18,27,173,176,177,179,185,186,188 \\
192,193)\end{array}$ & $(16,174,181,183)$ \\
\hline Oral ulcers & & $(18,27,181,184,194)$ & $(183)$ \\
\hline Serositis & $(17,18,175,178,181,182,184,187)$ & & $(174,183,186)$ \\
\hline Gastrointestinal complications & $(179)$ & & \\
\hline Renal disease & $\begin{array}{l}(17,18,27,174,176,177,185,187-191 \\
195,196)\end{array}$ & & $(16,181,183,186)$ \\
\hline Neurological and psychiatric disease & $\begin{array}{l}(182,185), \text { Seizure }(197), \text { peripheral } \\
\text { neuropathy }(17)\end{array}$ & Psychosis (177), psychiatric $(17,175)$ & $(181,183,186)$ \\
\hline $\begin{array}{l}\text { Hematological: thrombocytopenia, } \\
\text { leukopenia }\end{array}$ & $(18,27,177,195)$ & $(17,174,175,182,184,188,191)$ & $(181,183,186)$ \\
\hline Cardiovascular & $(27,176,179,187)$ & & $(181)$ \\
\hline Thromboses & $(27,179,180,190,193)$ & & \\
\hline Other & $\begin{array}{l}\text { Constitutional symptoms: fever, weight loss } \\
\text { (176), pleuritis (181), dry mouth and dry } \\
\text { eyes (185) }\end{array}$ & $\begin{array}{l}\text { Flares/severe flares (32); cutaneous (174); more } \\
\text { frequent relapses (17), erythrocyte sedimentation } \\
\text { rate, antinuclear antibody, anti-SSA, anti-SSB } \\
\text { (181) }\end{array}$ & $\begin{array}{l}\text { Mucocutaneous }(16,193) \text {, } \\
\text { vasculitis }(181,186) \text {, low C3, } \\
\text { anti-dsDNA, anti-Sm, anti- } \\
\text { rRNP }(181,193)\end{array}$ \\
\hline
\end{tabular}

Adapted with modifications from Ref. (15-18).

The results depict studies where significant $(P<0.05)$ differences were detected.

and autoimmune hepatitis), as well as a musculoskeletal $\mathrm{AD}$ (rheumatoid arthritis).

Although a strong female preponderance was observed with other ADs, these tend to vary with geographical locations. For example, antiphospholipid syndrome shows a female to male ratio of at least 3:1 worldwide, except in Spain and Italy, where it shows lower ratios (2.1:1 and 1.7:1, respectively). Immune thrombocytopenic purpura (ITP) was reported to have a $70 \%$ female prevalence in Denmark (213); however, the sex ratio is much lower in our dataset, ranging from 1.1:1 in the USA to 1.7:1 in France. Asian females seem to be more likely to get ITP than other ethnicities, with a female to male ratios ranging from 1.9:1 in India to 4.3:1 in Singapore. Other ADs reported include celiac disease (CoD) and ulcerative colitis (UC), which affected 57 and $65 \%$ of females, respectively (213). The female-to-male ratios for $\mathrm{CoD}$ and UC vary in our dataset, favoring males or females 
TABLE 4 | Comparative studies of male and female lupus: main clinical and demographic findings, adapted with modifications from Ref. (1, 8, 15-17).

\begin{tabular}{|c|c|c|c|c|c|c|c|}
\hline \multirow{2}{*}{$\begin{array}{l}\text { Year of } \\
\text { Study }\end{array}$} & \multirow[t]{2}{*}{ Country (ethnicity) } & \multirow[t]{2}{*}{ Study type } & \multirow[t]{2}{*}{ Size (\%male) } & \multirow[t]{2}{*}{ Age at onset } & \multicolumn{2}{|c|}{ Clinical phenotype $(P<0.05)$} & \multirow[t]{2}{*}{ Serology } \\
\hline & & & & & Increased in males & Increased in females & \\
\hline \multicolumn{8}{|c|}{ North America } \\
\hline NA & US (multiethnic) (173) & Prospective & $618(10.2)$ & $37.1(\mathrm{M}), 36.5(\mathrm{~F})$ & Renal disease & Musculoskeletal & $\operatorname{LAC}(\mathrm{M})$ \\
\hline 1969-1983 & US (198) & Inception & $361(17.2)$ & $44.7(\mathrm{M}), 35.2(\mathrm{~F})$ & Seizures & & \\
\hline 1982-1983 & US (175) & $\begin{array}{l}\text { Prospective } \\
\text { control }\end{array}$ & $100(50)$ & $45(\mathrm{M}), 44(\mathrm{~F})$ & Serositis & $\begin{array}{l}\text { Neurological, alopecia, } \downarrow \\
\text { platelets }\end{array}$ & \\
\hline 1987-2012 & US (multiethnic) (27) & Retrospective & 1979 (7.9) & $49.8(M), 37.6(F)$ & $\begin{array}{l}\text { Hypertension, renal disease, } \\
\text { Thrombotic episode, } \\
\text { hypertension, disability, } \\
\text { lymphopenia }\end{array}$ & $\begin{array}{l}\text { Malar rash, RP, } \\
\text { photosensitivity, } \\
\text { oral ulcers, } \\
\text { alopecia, arthralgia }\end{array}$ & $\begin{array}{l}\text { Anti-Sm, DAT, LAC, } \\
\text { anti-dsDNA, low } \\
\text { C3 (M) }\end{array}$ \\
\hline 2002-2007 & US (multiethnic) (195) & Case-control & $265(9)$ & NA & $\begin{array}{l}\text { Proteinuria, lymphopenia, } \\
\text { platelets count }\end{array}$ & & $\begin{array}{l}6 \text { antibodies } \\
\text { assayed, } P>0.05\end{array}$ \\
\hline \multicolumn{8}{|c|}{ Latin America } \\
\hline 1997-2005 & Latin America (176) & Inception & $1213(10.1)$ & $27(M), 29.2(F)$ & $\begin{array}{l}\text { Constitutional symptoms, } \\
\text { hypertension, proteinuria, } \\
\text { any renal, hemolytic anemia }\end{array}$ & $\begin{array}{l}\text { Arthralgia, alopecia, RP, } \\
\text { photosensitivity, any } \\
\text { cutaneous }\end{array}$ & $\begin{array}{l}\text { Low C3, } \\
\operatorname{lgG} \text { aCL (M) }\end{array}$ \\
\hline 1972-1993 & Latin America (190) & Cross-sectional & $1316(8.1)$ & $26(M), 28(F)$ & Renal disease & $\mathrm{RP}$ & dsDNA (M) \\
\hline 2000-2011 & $\begin{array}{l}\text { Colombia } \\
\text { (multiethnic) (16) }\end{array}$ & Cross-sectional & $160(25)$ & $32.0(\mathrm{M}), 30.5(\mathrm{~F})$ & Severe disease activity & Alopecia & anti-SSA/Ro (F) \\
\hline 2008-2012 & Brazil (189) & Prospective & $888(8.1)$ & $29.9(M), 29.9(F)$ & Malar rash, renal disease & & Anti-dsDNA (M) \\
\hline \multicolumn{8}{|c|}{ Scandinavia, Europe, and North Africa } \\
\hline 1980-1990 & Spanish (186) & Prospective & $261(11.5)$ & $34(\mathrm{M}), 31(\mathrm{~F})$ & $\begin{array}{l}\text { Discoid lesion, } \\
\text { subcutaneous lesion }\end{array}$ & Arthritis, malar rash & $\begin{array}{l}6 \text { antibodies } \\
\text { assayed, } P>0.05\end{array}$ \\
\hline $1981-2000$ & Greek (184) & Retrospective & $580(14)$ & $34.6(M), 31.4(F)$ & NA & $\begin{array}{l}\text { Photosensitivity, RP, oral } \\
\text { ulcers, anemia }\end{array}$ & NA \\
\hline 1982-2012 & UK (multiethnic) (194) & Retrospective & $484(9.3)$ & $30.9(\mathrm{M}), 29.1(\mathrm{~F})$ & NA & Oral ulcers & $\operatorname{lgM} \mathrm{aCL}(\mathrm{F})$ \\
\hline 1987-2006 & Spain (191) & Retrospective & $150(15.3)$ & $54(\mathrm{M}) 43(\mathrm{~F})$ & $\begin{array}{l}\text { Secondary Sjogren's } \\
\text { syndrome (over course of } \\
\text { disease), thrombocytopenia }\end{array}$ & $\mathrm{RP}$ & Anti-SSA/Ro(F) \\
\hline 1989-2007 & Greek (179) & Retrospective & $743(7.9)$ & $34(\mathrm{M}), 31(\mathrm{~F})$ & $\begin{array}{l}\text { Nephropathy, tendonitis, } \\
\text { myositis }\end{array}$ & NA & NA \\
\hline 1990-1999 & Tunisian (180) & Retrospective & $295(8.1)$ & NA & Vascular thrombosis & Alopecia & NA \\
\hline 1992-2006 & Spain (182) & Retrospective & $363(13)$ & $47.8(\mathrm{M}) 36.6(\mathrm{~F})$ & $\begin{array}{l}\text { Serositis, renal disease, } \\
\text { neurologic disorder }\end{array}$ & Leukopenia, alopecia & Anti-DNA (M) \\
\hline 2000-2008 & Danish (199) & Retrospective & $513(11.5)$ & $46.2(\mathrm{M}), 36.2(\mathrm{~F})$ & $\begin{array}{l}\text { Serositis, nephropathy, } \\
\text { hypertension }\end{array}$ & Photosensitivity & $\begin{array}{l}3 \text { antibodies } \\
\text { assayed, } P>0.05\end{array}$ \\
\hline \multicolumn{8}{|l|}{ Middle East } \\
\hline 1976-2011 & Iran (188) & Retrospective & $2355(10.1)$ & $25(\mathrm{M}), 24.5(\mathrm{~F})$ & Discoid rash, nephritis & Arthritis, leukopenia & \\
\hline 1996-2012 & $\begin{array}{l}\text { Turkey (185) } \\
\text { (Mediterranean) }\end{array}$ & Retrospective & $428(6.8)$ & $40.4(\mathrm{M}) 38.5(\mathrm{~F})$ & Renal disease, CNS & $\begin{array}{l}\text { Dry eyes, Dry mouth, } \\
\text { photosensitivity }\end{array}$ & \\
\hline \multicolumn{8}{|l|}{ Asia } \\
\hline 1990-1993 & Asian (192) & Retrospective & $147(41.5)$ & $28.2(\mathrm{M}), \mathrm{NA}(\mathrm{F})$ & NA & Arthritis, leukopenia & Anti-SSA/Ro (F) \\
\hline 1994-2010 & Korea (196) & Retrospective & $632(9)$ & $32.9(\mathrm{M}) 32.6(\mathrm{~F})$ & Renal disease & $\begin{array}{l}\text { Discoid rash, alopecia, } \\
\text { Leukopenia }\end{array}$ & Anti-SSA/Ro (F) \\
\hline 1999 & $\begin{array}{l}\text { HongKong (Asian) } \\
(32)\end{array}$ & $\begin{array}{l}\text { Retrospective } \\
\text { control }\end{array}$ & $252(20.2)$ & $31(\mathrm{M}), 31.9(\mathrm{~F})$ & NA & RP, alopecia & Anti-SSA/Ro (F) \\
\hline 2001 & $\begin{array}{l}\text { Malaysian (Asian) } \\
\text { (193) }\end{array}$ & Prospective & $134(9.0)$ & $30(\mathrm{M}), 26(\mathrm{~F})$ & Thrombosis & Arthritis & NA \\
\hline 2006-2010 & Indian (Asian) (183) & & $250(11.2)$ & $22.3(\mathrm{M}), 28.3(\mathrm{~F})$ & Renal disease & Disease severity & $\begin{array}{l}\text { Panel of } 13 \\
\text { antibodies, } \\
P>0.05\end{array}$ \\
\hline 2008 & Thai (Asian) (177) & $\begin{array}{l}\text { Retrospective } \\
\text { Case-control }\end{array}$ & 111 (33.3) & $34.6(\mathrm{M}), 34.4(\mathrm{~F})$ & $\begin{array}{l}\downarrow \text { Platelets, } \\
\uparrow \text { Serum creatinine }\end{array}$ & $\begin{array}{l}\text { Alopecia, arthralgia, RP, } \\
\text { psychosis }\end{array}$ & $\begin{array}{l}7 \text { antibodies } \\
\text { assayed, } P>0.05\end{array}$ \\
\hline 2010 & Chinese (181) & Retrospective & $1790(9.8)$ & $31.5(\mathrm{M}), 30.9(\mathrm{~F})$ & $\begin{array}{l}\text { Serositis, pleuritis, and } \\
\text { discoid rash }\end{array}$ & $\begin{array}{l}\text { Malar rash, alopecia, } \\
\text { oral ulcers, leukopenia } \\
\text { positively correlates } \\
\text { with age }\end{array}$ & $\begin{array}{l}\text { Elevated ESR, } \\
\text { antinuclear, } \\
\text { anti-SSA/Ro and } \\
\text { anti-SSB/La (F). } \\
\text { Anti-SSB/La } \\
\text { correlates with } \\
\text { age (M) }\end{array}$ \\
\hline
\end{tabular}

F, female; M, male; NA, not applicable; DAT, direct antiglobulin test; LAC, lupus anticoagulant; ESR, erythrocyte sedimentation rate; anti-dsDNA, anti-double stranded deoxyribonucleic acid; anti-Sm, anti-Smith; IgG Acl, anti-cardiolipin; anti-Sjögren's syndrome-related antigen A (SSARRo), anti- Sjögren's syndrome-related antigen B (SSB/La). 
TABLE 5 | Sex differences in ankylosing spondylitis clinical phenotypes.

\begin{tabular}{|c|c|c|c|}
\hline Clinical phenotype & $\begin{array}{c}\text { Studies showing phenotype } \\
\text { is significantly higher in } \\
\text { males }\end{array}$ & $\begin{array}{l}\text { Studies showing phenotype } \\
\text { is significantly higher in } \\
\text { females }\end{array}$ & $\begin{array}{l}\text { Studies showing statistically insignificant } \\
\text { differences in males and females }\end{array}$ \\
\hline \multicolumn{4}{|l|}{ Baseline characteristics } \\
\hline Age at onset & $(200)$ & $(141,201-204)$ & $(19)^{R(15)},(205,206)$ \\
\hline Age at diagnosis & & $(141,203,204)$ & $(19)^{R(15)},(202,206)$ \\
\hline Delay in diagnosis & & $(19)^{R(15)},(205)$ & $(141,203,204,206,207)$ \\
\hline Night pain & & $(206)$ & \\
\hline Sleep disturbance & & $(206)$ & \\
\hline Duration of morning stiffness & & $(207)$ & \\
\hline Relevant family history & & $(200,208,209)$ & $(202,203)$ \\
\hline HLA-B27-positive, \% & (202-204, 209) & & $(19,141,200,201,205)$ \\
\hline \multicolumn{4}{|c|}{ Disease activity and functional index } \\
\hline ESR & & $(201,206)$ & $(141,202,203,205,207)$ \\
\hline CRP & $(203-205,208)$ & $(201)$ & $(202,206,207)$ \\
\hline Disease activity: BASDAI score & & $(201,205-210)$ & $(141,202-204)$ \\
\hline BAS-G & & $(208)$ & $(203,205)$ \\
\hline Back pain & $(201,202,209)$ & $(203,205)$ & \\
\hline BASRI & $(207,209,210)$ & & \\
\hline BASRI-spinal & $(200,201)$ & & \\
\hline BASRI-hip & $(209)$ & & \\
\hline Physical function: BASFI score & & $(206,208)$ & $(141,200,201,203-205,207,209,210)$ \\
\hline Spinal mobility: BASMI score & $(203,204,207)$ & $(202)$ & $(205)$ \\
\hline Occiput-to-wall distance & $(202,207,209,210)$ & & \\
\hline Chest expansion & & & $(202,203,207,209,210)$ \\
\hline Modified Schober's test & & $(202,209,210)$ & $(207)$ \\
\hline Finger-to-floor & $(203,209,210)$ & & \\
\hline Lumbar rotation & & $(203)$ & \\
\hline
\end{tabular}

\section{Clinical data}

\section{MASES}

Enthesitis

Swollen joint score

Tender joint score

Definite deterioration and radiographic

progression-cervical spine

Cervical pain

Radiographic sacroiliitis, \%

Dactylitis

Root joint involvement (shoulder and hip)

Localization of clinical symptoms to buttock

Peripheral arthritis

Upper limb arthritis (\%)

Lower limb arthritis (\%)

Knee involvement

Intensity of axial pain

mSASSS

Thoracic syndesmophyte

Bamboo spine

Definite deterioration and radiographic

progression-lumbar spine

MRI-inflammatory lesions of the spine, \%

Uveitis

\section{Measures of Quality of Life}

SF-36 mental score

SF-36 physical score

ASQoL score

EuroQoL score

HAQ-AS
$(204,209)$

(202-208, 210)

(204, 208, 209)

(205, 207-209)

$(208,211)$

$(141,209)$

(205, 210)

(202)

(203, 209)

(208, 209)

$\begin{array}{lcr} & (209) & (204,210) \\ & (141) & (202,203,209) \\ & (208) & (141) \\ & (204,208) & (209) \\ (202) & (209) & (203) \\ (209) & & \\ (205) & (208) & (202) \\ (202) & (203) & (210) \\ (202) & & \\ (211) & & \\ (208) & (141,202) & \\ & & \\ & & \\ & & \\ & & \\ & & \end{array}$

The results depict studies where significant $(P<0.05)$ differences were detected; ${ }^{R(x)}$ indicates meta-analysis of $x$ number of published studies; ESR, erythrocyte sedimentation rate; CRP, C-reactive protein; BASDAl, Bath Ankylosing Spondylitis Disease Activity Index; BAS-G, Bath Ankylosing Spondylitis Patient Global disease activity score; BASRI, The Bath Ankylosing Spondylitis Radiology Index; BASFI, Bath Ankylosing Spondylitis Functional Index; BASMI, Bath Ankylosing Spondylitis Metrology Index; MASES, Maastricht Ankylosing Spondylitis Enthesitis Score; mSASSS, modified Stoke Ankylosing Spondylitis (AS) Spine Score; SF-36, Medical Outcomes Study 36-item Short Form; ASQoL, Ankylosing Spondylitis Quality of Life Questionnaire; EuroQoL, European Quality Of Life scale; HAQ-AS, Health Assessment Questionnaire for the Spondyloarthropathies. 
TABLE 6 | Comparative studies of male and female ankylosing spondylitis: main clinical and demographic findings.

\begin{tabular}{|c|c|c|c|c|c|c|c|}
\hline \multirow[t]{2}{*}{ Year of Study } & \multirow[t]{2}{*}{ Country (ethnicity) } & \multirow[t]{2}{*}{ Study type } & \multirow[t]{2}{*}{ Size (\% female) } & \multirow[t]{2}{*}{ Age at onset } & \multicolumn{2}{|c|}{ Clinical data $(P<0.05)$} & \multirow[t]{2}{*}{ Serology } \\
\hline & & & & & Higher in males & Higher in females & \\
\hline \multicolumn{8}{|l|}{ North America } \\
\hline 2007 & $\begin{array}{l}\text { USA (White, African } \\
\text { American, Asian/ } \\
\text { Pacific Islander, Native } \\
\text { American, Hispanic, } \\
\text { others) (200) }\end{array}$ & Prospective & $402(24.9)$ & $23.6(\mathrm{M}), 21.5(\mathrm{~F})$ & $\begin{array}{l}\text { BASRI, BASFI and } \\
\text { HAQ-S (when adjusted } \\
\text { for BASRI), thoracic, } \\
\text { and lumbar spinal } \\
\text { radiographic severity }\end{array}$ & $\begin{array}{l}\text { AS family history, neck and } \\
\text { peripheral joint pain }{ }^{*}\end{array}$ & NA \\
\hline
\end{tabular}

\section{Latin America} Brazil (209)

$1,505(27.6)$

Prospective $\quad 1,505(27.6)$
radiographic severity

2006-2009 \\ 2006-2009}

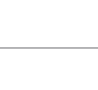

\author{
USA (White, African $\quad$ Prospective 402 (24.9) \\ ican, Hispanic,
}

NA

A

2006

2006

\author{
Argentina, Brazil, \\ Costa Rica, Chile, \\ Ecuador, Mexico, \\ Peru, Uruguay, and \\ Portugal (210)
}

\section{Europe}

2004-2009

UK (206)

Cross-sectional 1,072 (23.8)

NA

2005-2016 Switzerland (204)

Prospective $516(66.7)$

NA

Prospective $\quad 440$ (33.2)

$25(\mathrm{M}), 27.3(\mathrm{~F})$

$\%$ of HLA-B27+

patients, BASMI score

2004-2013 Spain (201)

2007-2010 France (208)

$\begin{array}{ll}\text { Retrospective } & 1,514(25.3) \\ \text { Prospective } & 475(49.7)\end{array}$

26.7 (M), 28.2 (F) Lumbalgia

NA

NA

\begin{tabular}{ll}
\hline 1996-2008 & $\begin{array}{l}\text { Netherlands, Belgium, Prospective } 216(62) \\
\text { France (205) }\end{array}$ \\
\hline
\end{tabular}

\section{Middle East and North Africa}

\begin{tabular}{rlll}
\hline 2010-2011 & $\begin{array}{l}\text { Iran (Fars, Turk, Kurd, } \\
\text { Lor, and others) (203) }\end{array}$ & Prospective & 320 (20.9)
\end{tabular}

$$
\text { Lor, and others) (203) }
$$

2009-2010 Morocco (207) $\quad$ Prospective $130(33.1) \quad 27.9$ (M), 28.8 (F)

\section{$\%$ of $\mathrm{HLA}-\mathrm{B} 27+$ patients, axial} inflammatory pain, lumbar pain, urethritis, occiput-to-wall and finger-to-floor distances,
BASRI, BASRI-spine, BASRIhip, grade 4 sacroilitis

BASRI, occiput-towall and finger-to-floor distance
AS family history, upper limb

NA arthritis, dactylitis and nail involvement, psoriasis, number of painful and swollen joints, MASES, BASDAI, ASQoL,

Schober's test

BASDAI, ASQoL, Enthesitis,

Schober's test

NA

$\rightarrow$ 


\begin{tabular}{|c|c|c|}
\hline Autoimmune Disease & $\begin{array}{c}\text { Female } \\
(\%)\end{array}$ & $\begin{array}{c}\text { Prevalence } \\
\left(\text { per } 10^{5}\right)\end{array}$ \\
\hline \multicolumn{3}{|c|}{ Neurological system } \\
\hline Multiple sclerosis & 64 & 58.3 \\
\hline Narcolepsy & 39 & 30.6 \\
\hline \multicolumn{3}{|c|}{ Endocrine system } \\
\hline Addison's disease & 63 & 14 \\
\hline Diabetes mellitus, type 1 & 45 & 480 \\
\hline Graves' disease & 88 & 629 \\
\hline Hashimoto's autoimmune thyroiditis & 95 & 791.7 \\
\hline \multicolumn{3}{|c|}{ Gastrointestinal system } \\
\hline Autoimmune hepatitis type 1 & 78 & 16.9 \\
\hline Coeliac disease & 57 & 750 \\
\hline Crohn's disease & 41 & 25 \\
\hline Pernicious anemia/atrophic gastritis & 67 & 150.9 \\
\hline Primary biliary cirrhosis & 89 & 14.6 \\
\hline Ulcerative colitis & 65 & 30 \\
\hline \multicolumn{3}{|c|}{ Haemopoetic system } \\
\hline Antiphospholipid syndrome & 74 & 21.5 \\
\hline Immune thrombocytopenic purpura & 70 & 72 \\
\hline \multicolumn{3}{|c|}{ Musculoskeletal system } \\
\hline Rheumatoid arthritis & 75 & 860 \\
\hline \multicolumn{3}{|c|}{ Cardiovascular system } \\
\hline Kawasaki disease & 40 & 10 \\
\hline Rheumatic fever & 50 & 250 \\
\hline Temporal arteritis & 85 & 30 \\
\hline \multicolumn{3}{|c|}{ Cutaneous/mucous membranes } \\
\hline Alopecia areata & 50 & 150 \\
\hline Dermatitis herpetiformis & 36 & 11.2 \\
\hline Vitiligo & 52 & 400.2 \\
\hline \multicolumn{3}{|l|}{ Systemic } \\
\hline Scleroderma & 92 & 24 \\
\hline Sjögren's syndrome & 94 & 14.4 \\
\hline Systemic lupus erythematosus & 88 & 32 \\
\hline
\end{tabular}

FIGURE 1 | List of Autoimmune Diseases (ADs) with prevalence $\geq 1$ per 10,000 people.

depending on geography. In Italy, UK, and India, males are more likely to suffer from $\mathrm{CoD}$ than females, whereas the reverse is true in Denmark and Sweden. In Asia, males are more likely to suffer from UC than in the Western world. AS and GBS show greater bias toward males, regardless of the geographical locations. In IPF, however, male predominance was observed all around the world except for the North Americas.

\section{ADs Have Different Trajectories in Different Sexes}

Autoimmune diseases are quintessentially immune system disorders. As the biology of the immune system varies between the two sexes, one would expect ADs to have different disease trajectories in males and females. Here, we utilize a female-biased AD: systemic lupus erythematosus (SLE), as well as a male-biased $\mathrm{AD}$ : ankylosing spondylitis (AS) to exemplify sex-bias in disease progression, complications, and mortality. SLE and AS data are stratified by clinical data, geography, and period of study, since older studies may suffer from delayed diagnoses in male SLE and female AS patients (Tables 3-6).
In SLE, clinical phenotypes show sex-bias. Alopecia, photosensitivity, malar rash, Raynaud's phenomenon, musculoskeletal complications, oral ulcers as well as psychiatric disorders are female-biased (Table 3 ). In addition, females are more likely to suffer from relapses and a concurrent diagnosis for SS. Male-specific clinical phenotypes include discoid lesions, serositis, renal disease, seizure, and peripheral neuropathy, as well as cardiovascular complications. Males also tend to display constitutional symptoms and higher score in Systemic Lupus Erythematosus Disease Activity Index, indicative of a higher disease activity. Indeed, higher mortality has been reported in male patients vs female patients. Serology in SLE also shows some sex-bias: females SLE patients more frequently present with higher erythrocyte sedimentation rate (ESR) and test positive for anti-SSA/Ro while the male SLE patients more frequently test positive for lupus anticoagulant, anti-Sm, anti-dsDNA, and hypocomplementemia.

In AS, clinical phenotypes also show some degree of sex-bias. Male AS patients tend to have disease onset at younger age and present with higher CRP, more back pain, knee involvement, higher scores for BASRI (including BASRI-spinal and BASRIhip), radiographic sacroilitis, higher modified Stoke Ankylosing Spondylitis Spinal Score, lower functional indices (occiput-towall and finger-to-floor distances), but higher SF-36 mental and physical scores. Female AS patients, on the other hand, present more with AS family history, higher Bath Ankylosing Spondylitis Disease Activity Index (BASDAI) score, enthesitis, more numbers of swollen, tender joints and peripheral arthritis but higher ASQoL score. Notably, in a multivariate model, Lee et al. found that for a given level of radiographic damage, female AS patients have more functional limitations than their male counterparts (200).

\section{DISCUSSION}

In this systematic review, we summarize sex differences in immune system biology, AD prevalence, as well as clinical phenotypes of SLE and AS. Data accrued highlight female predominance in common ADs, although there exist geographical differences in some cases. These observed geographical differences are suggestive of potential contributions of genetics and environmental factors toward $\mathrm{AD}$ pathology.

In SLE, disease complications and serology seem to show sexbias. Alopecia, for example, is exclusively observed in females just as serositis is exclusively seen in males. It is interesting how the serology in these males and females is reflective of sex-bias in clinical phenotypes. In The Genetic Profile Predicting the Phenotype (PROFILE) multiethnic cohort of 2,322 SLE patients, anti-Sm were significantly associated with antinuclear antibody, anti-double-stranded DNA (dsDNA), and clinical phenotypes, such as serositis, renal involvement, psychosis, vasculitis, Raynaud's phenomenon, hemolytic anemia, leukopenia, lymphopenia, and arterial hypertension (214). Furthermore, double positive serology for anti-Sm and anti-dsDNA has been strongly associated with renal involvement (215-218) and higher disease activity (219). Most of these clinical phenotypes are male-specific SLE complications (Tables 3 and 4). Similarly, anti-SSA/Ro antibodies have been reported to be strongly associated with low C3 (hypocomplementemia) and clinical phenotypes such 
as photosensitivity, subacute cutaneous lupus erythematosus, cutaneous vasculitis (palpable purpura), hematological disorder (anemia, leukopenia, and thrombocytopenia) (220-227), as well as Jaccoud's arthropathy (a type of arthritis) $(228,229)$. These phenotypes show female bias in our dataset (Tables 3 and 4). There are some clinical phenotypes such as mucocutaneous and hematological involvements, vasculitis, and association of antiSSA/Ro with low C3 that differ between these correlation studies and our dataset. This may arise either from ethnic or age differences in the different study groups, or the size of the study groups. The trend for renal involvement persists in all of the studies we have analyzed; however, this clinical phenotype may or may not show a statistical difference for sex-bias owing to the low number of male SLE patients in some studies.

We also observed sex-bias in our dataset for AS clinical phenotypes: female AS patients present with enthesitis and higher BASDAI scores, while male patients present with higher BASRI scores. AS is clinically tested with HLA-B27, ESR, and CRP. While some studies suggested that high CRP is more significantly seen in male patients and high ESR with female patients, many other studies have not come to similar conclusions.

The findings from SLE and AS suggest that disease phenotypes differ between males and females. In some cases, these diseases arguably have higher activity in the sex having lower prevalence. Awareness of sex-bias in disease presentation is crucial for early diagnosis, as well as treatment strategies for ADs in different sexes. More importantly, such awareness may guide the development of improved study design strategies for biomarker discovery.

\section{FUTURE DIRECTION AND CONCLUSION}

Timely diagnosis and treatment can be very effective for $\mathrm{AD}$ patients $(230,231)$ and biomarkers have great potential to enable it. Although AD biomarkers discovery is thriving, the same cannot be said of their clinical translation. Many biomarker projects fail at validation/replication stage (13) due to suboptimal sensitivity and specificity, as well as reproducibility in different studies (12). A few potential contributing factors to this observed failure include suboptimal infrastructure, study design, and execution in discovery-phase (12). Suboptimal study design includes small sample numbers, lack of patient history and subject matching (in terms of age, race, and sex) (12). Here we highlight the importance of sex stratification in biomarker discovery studies to promote reproducibility in replication/validation stage. Drawing example from SLE and AS, we note that differential clinical phenotypes exist in male and female patients. Different sexes may require different biomarkers for proper diagnosis of the same disease. From

\section{REFERENCES}

1. MacKay IR, Burnet M. Sir, 1899-1985, Autoimmune Diseases: Pathogenesis, Chemistry and Therapy. Springfield, IL: Thomas (1963).

2. Hueber W, Robinson WH. Proteomic biomarkers for autoimmune disease. Proteomics (2006) 6(14):4100-5. doi:10.1002/pmic.200600017

3. Klein SL, Flanagan KL. Sex differences in immune responses. Nat Rev Immunol (2016) 16(10):626-38. doi:10.1038/nri.2016.90

4. Fish EN. The X-files in immunity: sex-based differences predispose immune responses. Nat Rev Immunol (2008) 8(9):737-44. doi:10.1038/ nri2394
SLE serology we learn that some biomarkers are more frequently detected in one specific sex, and they show strong associations with sex-biased clinical phenotypes. Such specific associations may be missed when data from both sexes are aggregated.

In addition to enhancing sex-specific biomarker discovery and promoting reproducibility, a thorough understanding of sex differences in autoimmune milieu may guide disease prevention, diagnosis, and management. Our findings in Table 2 clearly demonstrate a higher prevalence ADs among females. Breast cancer screening mammography among women at average risk aged $50-74$ has been shown to reduce breast cancer mortality by $30-40 \%$ (232). These findings suggest potential benefits of AD screenings specifically for women, for early $\mathrm{AD}$ detection and reduction of mortality rates through early intervention. Another plausible area of further study is to sex-stratify serological benchmarks for males and females, in light of varying cytokine levels and activity in different sexes as observed in Table 1. We have limited our scope in this review to SLE, AS and sex stratification. Further stratifications for improved patient segmentation and more specific biomarker discovery may include stratifications by age, ethnicities and disease stages.

\section{AUTHOR CONTRIBUTIONS}

KP conceptualized, gathered literature for all other autoimmune diseases, consolidated literature review from others, and wrote the manuscript. JO gathered literature for IPF, SLE, gastrointestinal and liver autoimmune diseases and proofread the manuscript. SD gathered literature for autoimmune hepatitis. WT gathered literature for rheumatoid arthritis. NM gathered literature for ankylosing spondylitis and proofread the manuscript. JL gathered literature for SLE and antiphospholipid syndrome. CLD contributed to study design and provided clinical insights which enhanced manuscript quality.

\section{ACKNOWLEDGMENTS}

This project was supported by National Medical Research Council of Singapore (NMRC/CG/014/2013) and Agency for Science Technology and Research (A*STAR) Biomedical Research Council (SPF2014/001).

\section{SUPPLEMENTARY MATERIAL}

The Supplementary Material for this article can be found online at https://www.frontiersin.org/articles/10.3389/fimmu.2018.01208/ full\#supplementary-material.

5. Ngo ST, Steyn FJ, McCombe PA. Gender differences in autoimmune disease. Front Neuroendocrinol (2014) 35(3):347-69. doi:10.1016/j.yfrne.2014. 04.004

6. Fairweather D, Frisancho-Kiss S, Rose NR. Sex differences in autoimmune disease from a pathological perspective. Am J Pathol (2008) 173(3):600-9. doi:10.2353/ajpath.2008.071008

7. McCombe PA, Greer JM, Mackay IR. Sexual dimorphism in autoimmune disease. Curr Mol Med (2009) 9(9):1058-79. doi:10.2174/156652409789839116

8. Kinder JM, Stelzer IA, Arck PC, Way SS. Immunological implications of pregnancy-induced microchimerism, nature reviews. Immunology (2017) 17(8):483-94. doi:10.1038/nri.2017.38 
9. Marson A, Housley WJ, Hafler DA. Genetic basis of autoimmunity. J Clin Invest (2015) 125(6):2234-41. doi:10.1172/JCI78086

10. Floreani A, Leung PS, Gershwin ME. Environmental basis of autoimmunity. Clin Rev Allergy Immunol (2016) 50(3):287-300. doi:10.1007/ s12016-015-8493-8

11. Wizemann T, Pardue M; Institute of Medicine (US) Committee on Understanding the Biology of Sex and Gender Differences. Exploring the Biological Contributions to Human Health: Does Sex Matter? Washington, DC: National Academies Press (US) (2001).

12. Drucker E, Krapfenbauer K. Pitfalls and limitations in translation from biomarker discovery to clinical utility in predictive and personalised medicine. EPMA J (2013) 4(1):7. doi:10.1186/1878-5085-4-7

13. Willis JC, Lord GM. Immune biomarkers: the promises and pitfalls of personalized medicine. Nat Rev Immunol (2015) 15(5):323-9. doi:10.1038/ nri3820

14. Murphy KP. Janeway's Immunobiology. 8th ed. United States: Garland Science, Taylor \& Francis Group, LLC (2012).

15. Murphy G, Isenberg D. Effect of gender on clinical presentation in systemic lupus erythematosus. Rheumatology (2013) 52(12):2108-15. doi:10.1093/ rheumatology/ket160

16. Muñoz-Grajales C, González LA, Alarcón GS, Acosta-Reyes J. Gender differences in disease activity and clinical features in newly diagnosed systemic lupus erythematosus patients. Lupus (2016) 25(11):1217-23. doi:10.1177/0961203316635286

17. Yacoub Wasef SZ. Gender differences in systemic lupus erythematosus. Gend Med (2004) 1(1):12-7. doi:10.1016/S1550-8579(04)80006-8

18. Boodhoo KD, Liu S, Zuo X. Impact of sex disparities on the clinical manifestations in patients with systemic lupus erythematosus: a systematic review and meta-analysis. Medicine (Baltimore) (2016) 95(29):e4272. doi:10.1097/ MD. 0000000000004272

19. Jovani V, Blasco-Blasco M, Ruiz-Cantero MT, Pascual E. Understanding how the diagnostic delay of spondyloarthritis differs between women and men: a systematic review and metaanalysis. J Rheumatol (2017) 44(2):174-83. doi:10.3899/jrheum.160825

20. Gutcher I, Becher B. APC-derived cytokines and T cell polarization in autoimmune inflammation. JClin Invest (2007) 117(5):1119-27. doi:10.1172/ JCI31720

21. Ochiel DO, Rossoll RM, Schaefer TM, Wira CR. Effect of oestradiol and pathogen-associated molecular patterns on class II-mediated antigen presentation and immunomodulatory molecule expression in the mouse female reproductive tract. Immunology (2012) 135(1):51-62. doi:10.1111/j.1365-2567.2011.03512.x

22. Wira CR, Rossoll RM. Antigen-presenting cells in the female reproductive tract: influence of sex hormones on antigen presentation in the vagina. Immunology (1995) 84(4):505-8.

23. Lin AA, Wojciechowski SE, Hildeman DA. Androgens suppress antigen-specific $\mathrm{T}$ cell responses and IFN-gamma production during intracranial LCMV infection. J Neuroimmunol (2010) 226(1-2):8-19. doi:10.1016/j. jneuroim.2010.05.026

24. Huygen K, Palfliet K. Strain variation in interferon gamma production of BCG-sensitized mice challenged with PPD II. Importance of one major autosomal locus and additional sexual influences. Cell Immunol (1984) 85(1):75-81. doi:10.1016/0008-8749(84)90279-X

25. Amadori A, Zamarchi R, De Silvestro G, Forza G, Cavatton G, Danieli G, et al. Genetic control of the CD4/CD8 T-cell ratio in humans. Nat Med (1995) 1(12):1279-83. doi:10.1038/nm1295-1279

26. Feldman CH, Hiraki LT, Liu J, Fischer MA, Solomon DH, Alarcon GS, et al. Epidemiology and sociodemographics of systemic lupus erythematosus and lupus nephritis among US adults with Medicaid coverage, 2000-2004. Arthritis Rheum (2013) 65(3):753-63. doi:10.1002/art.37795

27. Tan TC, Fang H, Magder LS, Petri MA. Differences between male and female systemic lupus erythematosus in a multiethnic population. JRheumatol (2012) 39(4):759-69. doi:10.3899/jrheum.111061

28. Ji J, Sundquist J, Sundquist K. Gender-specific incidence of autoimmune diseases from national registers. J Autoimmun (2016) 69:102-6. doi:10.1016/ j.jaut.2016.03.003

29. Chiche L, Jourde N, Ulmann C, Mancini J, Darque A, Bardin N, et al. Seasonal variations of systemic lupus erythematosus flares in southern France. Eur J Intern Med (2012) 23(3):250-4. doi:10.1016/j.ejim.2011.12.006
30. Yong JLC, Killingsworth MC, Lai K. Renal biopsy pathology in a cohort of patients from southwest Sydney with clinically diagnosed systemic lupus erythematosus. Int J Nephrol Renovasc Dis (2013) 6:15-26. doi:10.2147/ IJNRD.S34357

31. Liu SS, Ye D, Lou J, Fan Z, Ye DQ. No evidence for a genetic association of IRF4 with systemic lupus erythematosus in a Chinese population. $Z$ Rheumatol (2014) 73(6):565-70. doi:10.1007/s00393-013-1279-6

32. Mok CC, Lau CS, Chan TM, Wong RW. Clinical characteristics and outcome of southern Chinese males with systemic lupus erythematosus. Lupus (1999) 8(3):188-96. doi:10.1191/096120399678847605

33. Shim JS, Sung YK, Joo YB, Lee HS, Bae SC. Prevalence and incidence of systemic lupus erythematosus in South Korea. Rheumatol Int (2014) 34(7):909-17. doi:10.1007/s00296-013-2915-9

34. Ohta A, Nagai M, Nishina M, Tomimitsu H, Kohsaka H. Age at onset and gender distribution of systemic lupus erythematosus, polymyositis/ dermatomyositis, and systemic sclerosis in Japan. Mod Rheumatol (2013) 23(4):759-64. doi:10.1007/s10165-012-0733-7

35. Nannini C, Jebakumar AJ, Crowson CS, Ryu JH, Matteson EL. Primary Sjögren's syndrome 1976-2005 and associated interstitial lung disease: a population-based study of incidence and mortality. BMJ Open (2013) 3(11): e003569. doi:10.1136/bmjopen-2013-003569

36. Eaton WW, Rose NR, Kalaydjian A, Pedersen MG, Mortensen PB. Epidemiology of autoimmune diseases in Denmark. J Autoimmun (2007) 29(1):1-9. doi:10.1016/j.jaut.2007.05.002

37. Seror R, GottenbergJE, Devauchelle-Pensec V, DubostJJ, Le Guern V, Hayem G, et al. European league against rheumatism Sjogren's syndrome disease activity index and European league against rheumatism Sjogren's syndrome patient-reported index: a complete picture of primary Sjogren's syndrome patients. Arthritis Care Res (2013) 65(8):1358-64. doi:10.1002/acr.21991

38. Diaz-Lopez C, Geli C, Corominas H, Malat N, Diaz-Torner C, Llobet JM, et al. Are there clinical or serological differences between male and female patients with primary Sjogren's syndrome? J Rheumatol (2004) 31(7): $1352-5$.

39. Downie-Doyle S, Bayat N, Rischmueller M, Lester S. Influence of CTLA4 haplotypes on susceptibility and some extraglandular manifestations in primary Sjogren's syndrome. Arthritis Rheum (2006) 54(8):2434-40. doi:10.1002/art.22004

40. Misra R, Hissaria P, Tandon V, Aggarwal A, Krishnani N, Dabadghao S. Primary Sjogren's syndrome: rarity in India. J Assoc Physicians India (2003) 51:859-62.

41. Tsuboi H, Asashima H, Takai C, Hagiwara S, Hagiya C, Yokosawa M, et al. Primary and secondary surveys on epidemiology of Sjögren's syndrome in Japan. Mod Rheumatol (2014) 24(3):464-70. doi:10.3109/14397595.2013. 843765

42. Zhao Y, Li Y, Wang L, Li X-F, Huang C-B, Wang G-C, et al. Primary Sjögren syndrome in Han Chinese: clinical and immunological characteristics of 483 patients. Medicine (2015) 94(16):e667. doi:10.1097/MD.0000000000000667

43. Raghu G, Weycker D, Edelsberg J, Bradford WZ, Oster G. Incidence and prevalence of idiopathic pulmonary fibrosis. Am J Respir Crit Care Med (2006) 174(7):810-6. doi:10.1164/rccm.200602-163OC

44. Raghu G, Chen SY, Yeh WS, Maroni B, Li Q, Lee YC, et al. Idiopathic pulmonary fibrosis in US Medicare beneficiaries aged 65 years and older: incidence, prevalence, and survival, 2001-11. Lancet Respir Med. (2014) 2(7):566-72. doi:10.1016/S2213-2600(14)70101-8

45. Hyldgaard C, Hilberg O, Muller A, Bendstrup E. A cohort study of interstitial lung diseases in central Denmark. Respir Med (2014) 108(5):793-9. doi:10.1016/j.rmed.2013.09.002

46. Ferrara G, Carlson L, Palm A, Einarsson J, Olivesten C, Skold M. Idiopathic pulmonary fibrosis in Sweden: report from the first year of activity of the Swedish IPF-registry. Eur Clin Respir J (2016) 3:31090. doi:10.3402/ecrj. v3.31090

47. Pedraza-Serrano F, López de Andrés A, Jiménez-García R, Jiménez-Trujillo I, Hernández-Barrera V, ánchez-Muñoz GS, et al. Retrospective observational study of trends in hospital admissions for idiopathic pulmonary fibrosis in Spain (2004-2013) using administrative data. BMJ Open (2017) 7(2):e013156. doi:10.1136/bmjopen-2016-013156

48. Gribbin J, Hubbard RB, Le Jeune I, Smith CJ, West J, Tata LJ. Incidence and mortality of idiopathic pulmonary fibrosis and sarcoidosis in the UK. Thorax (2006) 61(11):980-5. doi:10.1136/thx.2006.062836 
49. Behr J, Kreuter M, Hoeper MM, Wirtz H, Klotsche J, Koschel D, et al. Management of patients with idiopathic pulmonary fibrosis in clinical practice: the INSIGHTS-IPF registry. Eur Respir J (2015) 46(1):186-96. doi:10.1183/09031936.00217614

50. Moodley Y, Goh N, Glaspole I, Macansh S, Walters EH, Chapman S, et al. Australian Idiopathic Pulmonary Fibrosis Registry: vital lessons from a national prospective collaborative project. Respirology (2014) 19(7):1088-91. doi:10.1111/resp. 12358

51. Song JW, Hong SB, Lim CM, Koh Y, Kim DS. Acute exacerbation of idiopathic pulmonary fibrosis: incidence, risk factors and outcome. Eur Respir J (2011) 37(2):356. doi:10.1183/09031936.00159709

52. Natsuizaka M, Chiba H, Kuronuma K, Otsuka M, Kudo K, Mori M, et al. Epidemiologic survey of Japanese patients with idiopathic pulmonary fibrosis and investigation of ethnic differences. Am J Respir Crit Care Med (2014) 190(7):773-9. doi:10.1164/rccm.201403-0566OC

53. Gelber AC, Manno RL, Shah AA, Woods A, Le EN, Boin F, et al. Race and association with disease manifestations and mortality in scleroderma: a 20-year experience at the Johns Hopkins Scleroderma Center and review of the literature. Medicine (2013) 92(4):191-205. doi:10.1097/MD. 0b013e31829be125

54. Hoffmann-Vold AM, Midtvedt O, Molberg O, Garen T, Gran JT. Prevalence of systemic sclerosis in south-east Norway. Rheumatology (Oxford) (2012) 51(9):1600-5. doi:10.1093/rheumatology/kes076

55. Lo Monaco A, Bruschi M, La Corte R, Volpinari S, Trotta F. Epidemiology of systemic sclerosis in a district of northern Italy. Clin Exp Rheumatol (2011) 29(2 Suppl 65):S10-4.

56. Moinzadeh P, Fonseca C, Hellmich M, Shah AA, Chighizola C, Denton CP, et al. Association of anti-RNA polymerase III autoantibodies and cancer in scleroderma. Arthritis Res Ther (2014) 16(1):R53-53. doi:10.1186/ar4486

57. Strickland G, Pauling J, Cavill C, Shaddick G, McHugh N. Mortality in systemic sclerosis-a single centre study from the UK. Clin Rheumatol (2013) 32(10):1533-9. doi:10.1007/s10067-013-2289-0

58. Tolédano C, Gain M, Kettaneh A, Baudin B, Johanet C, Chérin P, et al. Aldolase predicts subsequent myopathy occurrence in systemic sclerosis. Arthritis Res Ther (2012) 14(3):R152-152. doi:10.1186/ar3888

59. Nikpour M, Hissaria P, Byron J, Sahhar J, Micallef M, Paspaliaris W, et al. Prevalence, correlates and clinical usefulness of antibodies to RNA polymerase III in systemic sclerosis: a cross-sectional analysis of data from an Australian cohort. Arthritis Res Ther (2011) 13(6):R211-211. doi:10.1186/ $\operatorname{ar} 3544$

60. Basappa K, Reddy KN. Period prevalence of systemic sclerosis (morphoea) in tertiary care hospital in India: an update. Am J Pharmtech Res (2013) 3(6): 368-74.

61. Pradhan V, Rajadhyaksha A, Nadkar M, Pandit P, Surve P, Lecerf M, et al. Clinical and autoimmune profile of scleroderma patients from Western India. Int J Rheumatol (2014) 2014:6. doi:10.1155/2014/983781

62. Yeung H, Takeshita J, Mehta NN, Kimmel SE, Ogdie A, Margolis DJ, et al. Psoriasis severity and the prevalence of major medical co-morbidities: a population-based study. JAMA Dermatol (2013) (10):1173-9. doi:10.1001/ jamadermatol.2013.5015

63. Takeshita J, Gelfand JM, Li P, Pinto L, Yu X, Rao P, et al. Psoriasis in the U.S. Medicare population: prevalence, treatment, and factors associated with biologic use. J Invest Dermatol (2015) 135(12):2955-63. doi:10.1038/ jid.2015.296

64. Danielsen K, Wilsgaard T, Olsen AO, Eggen AE, Olsen K, Cassano PA, et al. Elevated odds of metabolic syndrome in psoriasis: a population-based study of age and sex differences. Br J Dermatol (2015) 172(2):419-27. doi:10.1111/ bjd. 13288

65. Morales Suárez-Varela M, Reguera-Leal P, Grant WB, Rubio-López N, Llopis-González A. Vitamin D and psoriasis pathology in the Mediterranean region, Valencia (Spain). Int J Environ Res Public Health (2014) 11(12):1210817. doi:10.3390/ijerph111212108

66. Koch M, Baurecht H, Ried JS, Rodriguez E, Schlesinger S, Volks N, et al. Psoriasis and cardiometabolic traits: modest association but distinct genetic architectures. J Invest Dermatol (2015) 135(5):1283-93. doi:10.1038/jid.2015.8

67. Langan SM, Seminara NM, Shin DB, Troxel AB, Kimmel SE, Mehta NN, et al. Prevalence of metabolic syndrome in patients with psoriasis: a population-based study in the United Kingdom.J Invest Dermatol (2012) 132(3 Pt 1): 556-62. doi:10.1038/jid.2011.365
68. Plunkett A, Merlin K, Gill D, Zuo Y, Jolley D, Marks R. The frequency of common nonmalignant skin conditions in adults in central Victoria, Australia. Int J Dermatol (1999) 38(12):901-8. doi:10.1046/j.1365-4362.1999. 00856.x

69. Sakai R, Matsui S, Fukushima M, Yasuda H, Miyauchi H, Miyachi Y. Prognostic factor analysis for plaque psoriasis. Dermatology (2005) 211(2):103-6. doi:10.1159/000086437

70. Nerich V, Monnet E, Etienne A, Louafi S, Ramee C, Rican S, et al. Geographical variations of inflammatory bowel disease in France: a study based on national health insurance data. Inflamm Bowel Dis (2006) 12(3):218-26. doi:10.1097/01.MIB.0000206540.38834.8c

71. Vegh Z, Burisch J, Pedersen N, Kaimakliotis I, Duricova D, Bortlik M, et al. Incidence and initial disease course of inflammatory bowel diseases in 2011 in Europe and Australia: results of the 2011 ECCO-EpiCom inception cohort. J Crohns Colitis (2014) 8(11):1506-15. doi:10.1016/j.crohns.2014.06.004

72. Jess T, Riis L, Vind I, Winther KV, Borg S, Binder V, et al. Changes in clinical characteristics, course, and prognosis of inflammatory bowel disease during the last 5 decades: a population-based study from Copenhagen, Denmark. Inflamm Bowel Dis (2007) 13(4):481-9. doi:10.1002/ibd.20036

73. Asakura $\mathrm{K}$, Nishiwaki $\mathrm{Y}$, Inoue $\mathrm{N}$, Hibi $\mathrm{T}$, Watanabe $\mathrm{M}$, Takebayashi $\mathrm{T}$. Prevalence of ulcerative colitis and Crohn's disease in Japan. J Gastroenterol (2009) 44(7):659-65. doi:10.1007/s00535-009-0057-3

74. Rubio-Tapia A, Ludvigsson JF, Brantner TL, Murray JA, Everhart JE. The prevalence of celiac disease in the United States. Am J Gastroenterol (2012) 107(10):1538-44; quiz 1537, 1545. doi:10.1038/ajg.2012.219

75. Dixit R, Lebwohl B, Ludvigsson JF, Lewis SK, Rizkalla-Reilly N, Green PH. Celiac disease is diagnosed less frequently in young adult males. Dig Dis Sci (2014) 59(7):1509-12. doi:10.1007/s10620-014-3025-6

76. Jain AK, Sircar S, Jain M, Adkar S, Waghmare C. Inflammatory bowel disease in central India: a single centre experience over five years. Trop Doct (2012) 42(4):198-9. doi:10.1258/td.2012.120105

77. Ng SC, Tang W, Ching JY, Wong M, Chow CM, Hui AJ, et al. Incidence and phenotype of inflammatory bowel disease based on results from the Asia-Pacific Crohn's and colitis epidemiology study. Gastroenterology (2013) 145(1):158-65.e2. doi:10.1053/j.gastro.2013.04.007

78. Saleh MN, Fisher M, Grotzinger KM. Analysis of the impact and burden of illness of adult chronic ITP in the US. Curr Med Res Opin (2009) 25(12):2961-9. doi:10.1185/03007990903362388

79. Danese MD, Lindquist K, Gleeson M, Deuson R, Mikhael J. Cost and mortality associated with hospitalizations in patients with immune thrombocytopenic purpura. Am J Hematol (2009) 84(10):631-5. doi:10.1002/ajh. 21500

80. Boyle S, White RH, Brunson A, Wun T. Splenectomy and the incidence of venous thromboembolism and sepsis in patients with immune thrombocytopenia. Blood (2013) 121(23):4782-90. doi:10.1182/blood-2012$12-467068$

81. Frederiksen H, Maegbaek ML, Norgaard M. Twenty-year mortality of adult patients with primary immune thrombocytopenia: a Danish population-based cohort study. Br J Haematol (2014) 166(2):260-7. doi:10.1111/ bjh.12869

82. Grimaldi-Bensouda L, Nordon C, Michel M, Viallard JF, Adoue D, MagyBertrand N, et al. Immune thrombocytopenia in adults: a prospective cohort study of clinical features and predictors of outcome. Haematologica (2016) 101(9):1039-45. doi:10.3324/haematol.2016.146373

83. Winde G, Schmid KW, Lugering N, Fischer R, Brandt B, Berns T, et al. Results and prognostic factors of splenectomy in idiopathic thrombocytopenic purpura. J Am Coll Surg (1996) 183(6):565-74.

84. Schoonen WM, Kucera G, Coalson J, Li L, Rutstein M, Mowat F, et al. Epidemiology of immune thrombocytopenic purpura in the General Practice Research Database. Br J Haematol (2009) 145(2):235-44. doi:10.1111/ j.1365-2141.2009.07615.x

85. Seymour LA, Nourse JP, Crooks P, Wockner L, Bird R, Tran H, et al. The presence of KIR2DS5 confers protection against adult immune thrombocytopenia. Tissue Antigens (2014) 83(3):154-60. doi:10.1111/tan.12295

86. Nourse JP, Lea R, Crooks P, Wright G, Tran H, Catalano J, et al. The KIR2DS2/DL2 genotype is associated with adult persistent/chronic and relapsed immune thrombocytopenia independently of FCGR3a-158 polymorphisms. Blood Coagul Fibrinolysis (2012) 23(1):45-50. doi:10.1097/ MBC.0b013e32834d7ce3 
87. Choi PY, Gordon JE, Harvey M, Chong BH. Presentation and outcome of idiopathic thrombocytopenic purpura in a single Australian centre. Intern Med J (2012) 42(7):841-5. doi:10.1111/j.1445-5994.2012.02740.x

88. Zhou H, Fu R, Wang H, Zhou F, Li H, Zhou Z, et al. Immune thrombocytopenia in the elderly: clinical course in 525 patients from a single center in China. Ann Hematol (2013) 92(1):79-87. doi:10.1007/s00277-012-1567-2

89. Cheng G, Saleh MN, MarcherC, VaseyS, Mayer B, Aivado M, etal. Eltrombopag for management of chronic immune thrombocytopenia (RAISE): a 6-month, randomised, phase 3 study. Lancet (2011) 377(9763):393-402. doi:10.1016/ S0140-6736(10)60959-2

90. Rao K, Rao G, Patil N, Balaji O, Rao NR, Rao J, et al. A clinical study of patients with idiopathic thrombocytopenic purpura. Asian J Pharm Clin Res (2017) 10(7):373-77. doi:10.22159/ajpcr.2017.v10i7.18775

91. Kueh YK. Adult idiopathic thrombocytopenic purpura (ITP) - a prospective tracking of its natural history. Singapore Med J (1995) 36:367-70.

92. Phitayakorn R, Morales-Garcia D, Wanderer J, Lubitz CC, Gaz RD, Stephen AE, et al. Surgery for Graves' disease: a 25-year perspective. Am J Surg (2013) 206(5):669-73. doi:10.1016/j.amjsurg.2013.07.005

93. Carle A, Pedersen IB, Knudsen N, Perrild H, Ovesen L, Rasmussen LB, et al. Epidemiology of subtypes of hyperthyroidism in Denmark: a population-based study. Eur J Endocrinol (2011) 164(5):801-9. doi:10.1530/ EJE-10-1155

94. Krassas GE, Tziomalos K, Pontikides N, Lewy H, Laron Z. Seasonality of month of birth of patients with Graves' and Hashimoto's diseases differ from that in the general population. Eur J Endocrinol (2007) 156(6):631-6. doi:10.1530/EJE-07-0015

95. Gaujoux S, Leenhardt L, Trésallet C, Rouxel A, Hoang C, Jublanc C, et al. Extensive thyroidectomy in Graves' disease. J Am Coll Surg (2006) 202(6):868-73. doi:10.1016/j.jamcollsurg.2006.02.031

96. Khong JJ, Finch S, De Silva C, Rylander S, Craig JE, Selva D, et al. Risk factors for Graves' orbitopathy; the Australian thyroid-associated orbitopathy research (ATOR) study. J Clin Endocrinol Metab (2016) 101(7):2711-20. doi:10.1210/jc.2015-4294

97. Guo T, Huo YN, Zhu W, Xu F, Liu C, Liu N, et al. Genetic association between IL-17F gene polymorphisms and the pathogenesis of Graves' Disease in the Han Chinese population. Gene (2013) 512(2):300-4. doi:10.1016/j.gene. 2012.10.021

98. Staii A, Mirocha S, Todorova-Koteva K, Glinberg S, Jaume JC. Hashimoto thyroiditis is more frequent than expected when diagnosed by cytology which uncovers a pre-clinical state. Thyroid Res (2010) 3:11-11. doi:10.1186/ 1756-6614-3-11

99. Tjiang H, Lahooti H, McCorquodale T, Parmar KR, Wall JR. Eye and eyelid abnormalities are common in patients with Hashimoto's thyroiditis. Thyroid (2010) 20(3):287-90. doi:10.1089/thy.2009.0199

100. Zhang L, Li H, Ji Q-H, Zhu Y-X, Wang Z-Y, Wang Y, et al. The clinical features of papillary thyroid cancer in Hashimoto's thyroiditis patients from an area with a high prevalence of Hashimoto's disease. BMC Cancer (2012) 12(1):610. doi:10.1186/1471-2407-12-610

101. Sahoo JP, Selviambigapathy J, Kamalanathan S, Negi VS, Sridhar MG, Kar SS, et al. The Serological and biochemical markers of adrenal cortex and endocrine pancreas dysfunction in patients with Hashimoto's thyroiditis: a hospital-based pilot study. Ind J Endocrinol Metab (2017) 21(4):540-4. doi:10.4103/ijem.IJEM_72_17

102. Costacou T, Fried L, Ellis D, Orchard TJ. Sex differences in the development of kidney disease in individuals with type 1 diabetes mellitus: a contemporary analysis. Am J Kidney Dis (2011) 58(4):565-73. doi:10.1053/ j.ajkd.2011.05.025

103. Gale EA, Gillespie KM. Diabetes and gender. Diabetologia (2001) 44(1):3-15. doi: $10.1007 /$ s001250051573

104. Kyvik KO, Nystrom L, Gorus F, Songini M, Oestman J, Castell C, et al. The epidemiology of type 1 diabetes mellitus is not the same in young adults as in children. Diabetologia (2004) 47(3):377-84. doi:10.1007/ s00125-004-1331-9

105. Huxley RR, Peters SA, Mishra GD, Woodward M. Risk of all-cause mortality and vascular events in women versus men with type 1 diabetes: a systematic review and meta-analysis. Lancet Diabetes Endocrinol (2015) 3(3):198-206. doi:10.1016/S2213-8587(14)70248-7

106. Speight J, Browne JL, Holmes-Truscott E, Hendrieckx C, Pouwer F. Diabetes MILES - Australia (management and impact for long-term empowerment and success): methods and sample characteristics of a national survey of the psychological aspects of living with type 1 or type 2 diabetes in Australian adults. BMC Public Health (2012) 12:120. doi:10.1186/1471-245812-120

107. Bhadada SK, Kochhar R, Bhansali A, Dutta U, Kumar PR, Poornachandra KS, et al. Prevalence and clinical profile of celiac disease in type 1 diabetes mellitus in north India. J Gastroenterol Hepatol (2011) 26(2):378-81. doi:10.1111/j.1440-1746.2010.06508.x

108. Kawasaki E, Eguchi K. Is type 1 diabetes in the Japanese population the same as among Caucasians? Ann N Y Acad Sci (2004) 1037:96-103. doi:10.1196/ annals.1337.014

109. Parikh-Patel A, Gold EB, Worman H, Krivy KE, Gershwin ME. Risk factors for primary biliary cirrhosis in a cohort of patients from the united states. Hepatology (2001) 33(1):16-21. doi:10.1053/jhep.2001.21165

110. Gershwin ME, Selmi C, Worman HJ, Gold EB, Watnik M, Utts J, et al. Risk factors and comorbidities in primary biliary cirrhosis: a controlled interview-based study of 1032 patients. Hepatology (2005) 42(5):1194-202. doi:10.1002/hep.20907

111. Corpechot C, Chretien Y, Chazouilleres O, Poupon R. Demographic, lifestyle, medical and familial factors associated with primary biliary cirrhosis. J Hepatol (2010) 53(1):162-9. doi:10.1016/j.jhep.2010.02.019

112. Prince MI, Ducker SJ, James OFW. Case-control studies of risk factors for primary biliary cirrhosis in two United Kingdom populations. Gut (2010) 59(4):508. doi:10.1136/gut.2009.184218

113. Sood S, Gow PJ, Christie JM, Angus PW. Epidemiology of primary biliary cirrhosis in Victoria, Australia: high prevalence in migrant populations. Gastroenterology (2004) 127(2):470-5. doi:10.1053/j.gastro.2004. 04.064

114. Wang L, Zhang F-C, Chen H, Zhang X, Xu D, Li Y-Z, et al. Connective tissue diseases in primary biliary cirrhosis: a population-based cohort study. World J Gastroenterol (2013) 19(31):5131-7. doi:10.3748/wjg.v19. i31.5131

115. Ikeda F, Okamoto R, Baba N, Fujioka S, Shoji B, Yabushita K, et al. Prevalence and associated factors with esophageal varices in early primary biliary cirrhosis. J Gastroenterol Hepatol (2012) 27(8):1320-8. doi:10.1111/j.1440-1746. 2012.07114.x

116. Wong RJ, Gish R, Frederick T, Bzowej N, Frenette C. The impact of race/ ethnicity on the clinical epidemiology of autoimmune hepatitis. J Clin Gastroenterol (2012) 46(2):155-61. doi:10.1097/MCG.0b013e318228b781

117. Werner M, Prytz H, Ohlsson B, Almer S, Bjornsson E, Bergquist A, et al. Epidemiology and the initial presentation of autoimmune hepatitis in Sweden: a nationwide study. Scand J Gastroenterol (2008) 43(10):1232-40. doi:10.1080/00365520802130183

118. Boberg KM, Aadland E, Jahnsen J, Raknerud N, Stiris M, Bell H. Incidence and prevalence of primary biliary cirrhosis, primary sclerosing cholangitis, and autoimmune hepatitis in a Norwegian population. Scand J Gastroenterol (1998) 33(1):99-103. doi:10.1080/00365529850166284

119. Gronbaek L, Vilstrup H, Jepsen P. Autoimmune hepatitis in Denmark: incidence, prevalence, prognosis, and causes of death. A nationwide registry-based cohort study. J Hepatol (2014) 60(3):612-7. doi:10.1016/j. jhep.2013.10.020

120. Primo J, Merino C, Fernandez J, Moles JR, Llorca P, Hinojosa J. [Incidence and prevalence of autoimmune hepatitis in the area of the Hospital de Sagunto (Spain)]. Gastroenterol Hepatol (2004) 27(4):239-43. doi:10.1016/ S0210-5705(03)70452-X

121. Muratori P, Czaja AJ, Muratori L, Pappas G, Maccariello S, Cassani F, et al. Genetic distinctions between autoimmune hepatitis in Italy and North America. World J Gastroenterol (2005) 11(12):1862-6. doi:10.3748/wjg.v11. i12.1862

122. Vogel A, Strassburg CP, Manns MP. Genetic association of vitamin D receptor polymorphisms with primary biliary cirrhosis and autoimmune hepatitis. Hepatology (2002) 35(1):126-31. doi:10.1053/jhep.2002.30084

123. Ngu JH, Bechly K, Chapman BA, Burt MJ, Barclay ML, Gearry RB, et al. Population-based epidemiology study of autoimmune hepatitis: a disease of older women? J Gastroenterol Hepatol (2010) 25(10):1681-6. doi:10.1111/j.1440-1746.2010.06384.x

124. Haider AS, Kaye G, Thomson A. Autoimmune hepatitis in a demographically isolated area of Australia. Intern Med J (2010) 40(4):281-5. doi:10.1111/j.1445-5994.2009.02041.x 
125. Miyake Y, Iwasaki Y, Kobashi H, Yasunaka T, Ikeda F, Takaki A, et al. Autoimmune hepatitis with acute presentation in Japan. Dig Liver Dis (2010) 42(1):51-4. doi:10.1016/j.dld.2009.04.009

126. Peng M, Li Y, Zhang M, Jiang Y, Xu Y, Tian Y, et al. Clinical features in different age groups of patients with autoimmune hepatitis. Exp Ther Med (2014) 7(1):145-8. doi:10.3892/etm.2013.1363

127. Choudhuri G, Somani SK, Baba CS, Alexander G. Autoimmune hepatitis in India: profile of an uncommon disease. BMC Gastroenterol (2005) 5(1):27. doi:10.1186/1471-230X-5-27

128. Lee YM, Teo EK, Ng TM, Khor C, Fock KM. Autoimmune hepatitis in Singapore: a rare syndrome affecting middle-aged women. J Gastroenterol Hepatol (2001) 16(12):1384-9. doi:10.1046/j.1440-1746.2001.02646.x

129. Gathungu G, Zhang CK, Zhang W, Cho JH. A two-marker haplotype in the IRF5 gene is associated with inflammatory bowel disease in a North American cohort. Genes Immun (2012) 13(4):351-5. doi:10.1038/gene.2011.90

130. Mustalahti K, Catassi C, Reunanen A, Fabiani E, Heier M, McMillan S, et al. The prevalence of celiac disease in Europe: results of a centralized, international mass screening project. Ann Med (2010) 42(8):587-95. doi:10.3109/ 07853890.2010 .505931

131. Ronnblom A, Holmstrom T, Tanghoj H, Wanders A, Sjoberg D. Celiac disease, collagenous sprue and microscopic colitis in IBD. Observations from a population-based cohort of IBD (ICURE). Scand J Gastroenterol (2015) 50(10):1234-40. doi:10.3109/00365521.2015.1041152

132. Cook HB, Burt MJ, Collett JA, Whitehead MR, Frampton CM, Chapman BA. Adult coeliac disease: prevalence and clinical significance. J Gastroenterol Hepatol (2000) 15(9):1032-6. doi:10.1046/j.1440-1746.2000.02290.x

133. Nijhawan S, Katiyar P, Nagaich N, Saradava V, Nijhawan M, Gupta G, et al. Prevalence of associated disorders in Indian patients with celiac disease. Ind J Gastroenterol (2013) 32(5):330-4. doi:10.1007/s12664-013-0345-y

134. Wu J, Xia B, von Blomberg BM, Zhao C, Yang XW, Crusius JB, et al. Coeliac disease: emerging in China? Gut (2010) 59(3):418-9. doi:10.1136/ gut.2009.197863

135. Carter ET, McKenna CH, Brian DD, Kurland LT. Epidemiology of ankylosing spondylitis in Rochester, Minnesota, 1935-1973. Arthritis Rheum (1979) 22(4):365-70. doi:10.1002/art.1780220408

136. Kaipiainen-Seppanen $\mathrm{O}$, Aho K. Incidence of chronic inflammatory joint diseases in Finland in 1995. J Rheumatol (2000) 27(1):94-100.

137. Haglund E, Bremander AB, Petersson IF, StrombeckB, Bergman S, Jacobsson LT, et al. Prevalence of spondyloarthritis and its subtypes in southern Sweden. Ann Rheum Dis (2011) 70(6):943-8. doi:10.1136/ard.2010.141598

138. TrontzasP,AndrianakosA,MiyakisS, PantelidouK, VafiadouE, GarantziotouV, et al. Seronegative spondyloarthropathies in Greece: a population-based study of prevalence, clinical pattern, and management. The ESORDIG study. Clin Rheumatol (2005) 24(6):583-9. doi:10.1007/s10067-005-1106-9

139. Oldroyd J, Schachna L, Buchbinder R, Staples M, Murphy B, Bond M, et al. Ankylosing spondylitis patients commencing biologic therapy have high baseline levels of comorbidity: a report from the Australian Rheumatology Association Database. Int J Rheumatol (2009) 2009:268569. doi:10.1155/2009/268569

140. Xiang Y-J, Dai S-M. Prevalence of rheumatic diseases and disability in China. Rheumatol Int (2008) 29(5):481. doi:10.1007/s00296-008-0809-z

141. Aggarwal R, Malaviya AN. Clinical characteristics of patients with ankylosing spondylitis in India. Clin Rheumatol (2009) 28(10):1199-205. doi:10.1007/ s10067-009-1227-7

142. Hukuda S, Minami M, Saito T, Mitsui H, Matsui N, Komatsubara Y, et al. Spondyloarthropathies in Japan: nationwide questionnaire survey performed by the Japan Ankylosing Spondylitis Society. J Rheumatol (2001) 28(3):554-9.

143. Gonzalez A, Maradit Kremers H, Crowson CS, Nicola PJ, Davis JM III, Therneau TM, et al. The widening mortality gap between rheumatoid arthritis patients and the general population. Arthritis Rheum (2007) 56(11):3583-7. doi:10.1002/art.22979

144. Humphreys JH, Verstappen SMM, Hyrich KL, Chipping JR, Marshall T, Symmons DPM. The incidence of rheumatoid arthritis in the UK: comparisons using the 2010 ACR/EULAR classification criteria and the 1987 ACR classification criteria. Results from the Norfolk Arthritis Register. Ann Rheum Dis (2013) 72(8):1315-20. doi:10.1136/annrheumdis-2012-201960

145. Wilson FC, Icen M, Crowson CS, McEvoy MT, Gabriel SE, Kremers HM. Time trends in epidemiology and characteristics of psoriatic arthritis over
3 decades: a population-based study. J Rheumatol (2009) 36(2):361-7. doi:10.3899/jrheum.080691

146. Madland TM, Apalset EM, Johannessen AE, Rossebo B, Brun JG. Prevalence, disease manifestations, and treatment of psoriatic arthritis in Western Norway. J Rheumatol (2005) 32(10):1918-22.

147. Hadjimichael O, Vollmer T, Oleen-Burkey M; North American Research Committee on Multiple Sclerosis. Fatigue characteristics in multiple sclerosis: the North American Research Committee on Multiple Sclerosis (NARCOMS) survey. Health Qual Life Outcomes (2008) 6(1):100. doi:10.1186/1477-7525-6-100

148. Bentzen J, Meulengracht Flachs E, Stenager E, Brønnum-Hansen H, KochHenriksen N. Prevalence of multiple sclerosis in Denmark 1950-2005. Mult Scler J (2010) 16(5):520-5. doi:10.1177/1352458510364197

149. El Adssi H, Debouverie M, Guillemin F. Estimating the prevalence and incidence of multiple sclerosis in the Lorraine region, France, by the capture-recapture method. Mult Scler J (2012) 18(9):1244-50. doi:10.1177/ 1352458512437811

150. Hammond SR, de Wytt C, Maxwell IC, Landy PJ, English D, McLeod JG, et al. The epidemiology of multiple sclerosis in Queensland, Australia. J Neurol Sci (1987) 80(2):185-204. doi:10.1016/0022-510X(87)90154-7

151. O'Gorman C, Bukhari W, Todd A, Freeman S, Broadley SA. Smoking increases the risk of multiple sclerosis in Queensland, Australia. J Clin Neurosci (2014) 21(10):1730-3. doi:10.1016/j.jocn.2014.01.009

152. Cheng Q, Cheng XJ, Jiang GX. Multiple sclerosis in China-history and future. Mult Scler (2009) 15(6):655-60. doi:10.1177/1352458509102921

153. Osoegawa M, Kira J, Fukazawa T, Fujihara K, Kikuchi S, Matsui M, et al. Temporal changes and geographical differences in multiple sclerosis phenotypes in Japanese: nationwide survey results over 30 years. Mult Scler (2008) 15(2):159-73. doi:10.1177/1352458508098372

154. Pandit L, Kundapur R. Prevalence and patterns of demyelinating central nervous system disorders in urban Mangalore, South India. Mult Scler (2014) 20(12):1651-3. doi:10.1177/1352458514521503

155. Guptill JT, Marano A, Krueger A, Sanders DB. Cost analysis of myasthenia gravis from a large U.S. insurance database. Muscle Nerve (2011) 44(6): 907-11. doi:10.1002/mus.22212

156. Mantegazza R, Baggi F, Antozzi C, Confalonieri P, Morandi L, Bernasconi P, et al. Myasthenia gravis (MG): epidemiological data and prognostic factors. Ann N Y Acad Sci (2003) 998:413-23. doi:10.1196/annals.1254.054

157. Guy-Coichard C, Nguyen DT, Delorme T, Boureau F. Pain in hereditary neuromuscular disorders and myasthenia gravis: a national survey of frequency, characteristics, and impact. J Pain Symptom Manage (2008) 35(1):40-50. doi:10.1016/j.jpainsymman.2007.02.041

158. Gattellari M, Goumas C, Worthington JM. A national epidemiological study of myasthenia gravis in Australia. Eur J Neurol (2012) 19(11):1413-20. doi:10.1111/j.1468-1331.2012.03698.x

159. Suzuki Y, Utsugisawa K, Suzuki S, Nagane Y, Masuda M, Kabasawa C, et al. Factors associated with depressive state in patients with myasthenia gravis: a multicentre cross-sectional study. BMJ Open (2011) 1(2):e000313. doi:10.1136/bmjopen-2011-000313

160. Singhal B, Bhatia N, Umesh T, Menon S. Myasthenia gravis: a study from India. Neurol India (2008) 56(3):352-5. doi:10.4103/0028-3886.43455

161. Huang X, Liu WB, Men LN, Feng HY, Li Y, Luo CM, et al. Clinical features of myasthenia gravis in southern China: a retrospective review of 2,154 cases over 22 years. Neurol Sci (2013) 34(6):911-7. doi:10.1007/s10072-012$1157-z$

162. Alshekhlee A, Hussain Z, Sultan B, Katirji B. Guillain-Barre syndrome: incidence and mortality rates in US hospitals. Neurology (2008) 70(18):1608-13. doi:10.1212/01.wnl.0000310983.38724.d4

163. Sipila JO, Soilu-Hanninen M. The incidence and triggers of adult-onset Guillain-Barre syndrome in southwestern Finland 2004-2013. Eur J Neurol (2015) 22(2):292-8. doi:10.1111/ene.12565

164. Forsberg A, de Pedro-Cuesta J, Widen Holmqvist L. Use of healthcare, patient satisfaction and burden of care in Guillain-Barre syndrome. J Rehabil Med (2006) 38(4):230-6. doi:10.1080/16501970600582997

165. Benedetti MD, Pugliatti M, D’Alessandro R, Beghi E, Chio A, Logroscino G et al. Incidence study of Guillain-Barre syndrome in Italy. The ITANG Study. Neuroepidemiology (2015) 45(2):90-9. doi:10.1159/000438752

166. Webb AJ, Brain SA, Wood R, Rinaldi S, Turner MR. Seasonal variation in Guillain-Barre syndrome: a systematic review, meta-analysis and Oxfordshire 
cohort study. J Neurol Neurosurg Psychiatry (2015) 86(11):1196-201. doi:10.1136/jnnp-2014-309056

167. Pouwels S, de Boer A, Leufkens HGM, Weber WEJ, Cooper C, van Staa TP, et al. Risk of fracture in patients with Guillain-Barré syndrome. Osteoporos Int (2014) 25(7):1845-51. doi:10.1007/s00198-013-2442-2

168. Blum S, Reddel S, Spies J, McCombe P. Clinical features of patients with Guillain-Barre syndrome at seven hospitals on the East Coast of Australia. J Peripher Nerv Syst (2013) 18(4):316-20. doi:10.1111/jns5.12045

169. Dhadke SV, Dhadke VN, Bangar SS, Korade MB. Clinical profile of Guillain Barre syndrome. J Assoc Physicians India (2013) 61(3):168-72.

170. Cheng Q, Wang DS, Jiang GX, Han H, Zhang Y, Wang WZ, et al. Distinct pattern of age-specific incidence of Guillain-Barre syndrome in Harbin, China. J Neurol (2002) 249(1):25-32. doi:10.1007/PL00007844

171. Mitsui Y, Kusunoki S, Arimura K, Kaji R, Kanda T, Kuwabara S, et al. A multicentre prospective study of Guillain-Barre syndrome in Japan: a focus on the incidence of subtypes. J Neurol Neurosurg Psychiatry (2015) 86(1):110-4. doi:10.1136/jnnp-2013-306509

172. Prete PE, Majlessi A, Gilman S, Hamideh F. Systemic lupus erythematosus in men: a retrospective analysis in a Veterans Administration Healthcare System population. J Clin Rheumatol (2001) 7(3):142-50. doi:10.1097/00124743200106000-00003

173. Rivest C, Lew RA, Welsing PM, Sangha O, Wright EA, Roberts WN, et al. Association between clinical factors, socioeconomic status, and organ damage in recent onset systemic lupus erythematosus. J Rheumatol (2000) 27(3):680-4.

174. de Carvalho JF, do Nascimento AP, Testagrossa LA, Barros RT, Bonfa E. Male gender results in more severe lupus nephritis. Rheumatol Int (2010) 30(10):1311-5. doi:10.1007/s00296-009-1151-9

175. Miller MH, Urowitz MB, Gladman DD, Killinger DW. Systemic lupus erythematosus in males. Medicine (Baltimore) (1983) 62(5):327-34. doi:10.1097/00005792-198309000-00005

176. Garcia MA, Marcos JC, Marcos AI, Pons-Estel BA, Wojdyla D, Arturi A, et al. Male systemic lupus erythematosus in a Latin-American inception cohort of 1214 patients. Lupus (2005) 14(12):938-46. doi:10.1191/0961203305lu2245oa

177. Mongkoltanatus J, Wangkaew S, Kasitanon N, Louthrenoo W. Clinical features of Thai male lupus: an age-matched controlled study. Rheumatol Int (2008) 28(4):339-44. doi:10.1007/s00296-007-0442-2

178. Keskin G, Tokgoz G, Duzgun N, Duman M, Kinikli G, Olmez U, et al. Systemic lupus erythematosus in Turkish men. Clin Exp Rheumatol (2000) 18(1):114-5.

179. Stefanidou S, Benos A, Galanopoulou V, Chatziyannis I, Kanakoudi F, Aslanidis S, et al. Clinical expression and morbidity of systemic lupus erythematosus during a post-diagnostic 5-year follow-up: a male:female comparison. Lupus (2011) 20(10):1090-4. doi:10.1177/0961203311403640

180. Othmani S, Louzir B; Group d'etude du lupus. [Systemic lupus erythematosus in 24 tunisian males: clinico-biological analysis and clinical course]. La Revue de medecine interne (2002) 23(12):983-90. doi:10.1016/S0248-8663(02) 00684-7

181. Feng J-B, Ni J-D, Yao X, Pan H-F, Li X-P, Xu J-H, et al. Gender and age influence on clinical and laboratory features in Chinese patients with systemic lupus erythematosus: 1,790 cases. Rheumatol Int (2010) 30(8):1017-23. doi:10.1007/s00296-009-1087-0

182. Gomez J, Suarez A, Lopez P, Mozo L, Diaz JB, Gutierrez C. Systemic lupus erythematosus in Asturias, Spain: clinical and serologic features. Medicine (2006) 85(3):157-68. doi:10.1097/01.md.0000224711.54886.b1

183. Patwardhan M, Pradhan V, Rajadhyaksha A, Umare V, Rajendran V, Surve P, et al. Clinical and serological features of male systemic lupus erythematosus patients from Western India. Ind J Rheumatol (2012) 7(4):204-8. doi:10.1016/j.injr.2012.09.002

184. Voulgari PV, Katsimbri P, Alamanos Y, Drosos AA. Gender and age differences in systemic lupus erythematosus. A study of 489 Greek patients with a review of the literature. Lupus (2002) 11(11):722-9. doi:10.1191/0961203 302lu253oa

185. Pamuk ON, Akbay FG, Donmez S, Yilmaz N, Calayir GB, Yavuz S. The clinical manifestations and survival of systemic lupus erythematosus patients in Turkey: report from two centers. Lupus (2013) 22(13):1416-24. doi:10.1177/0961203313499956

186. Font J, Cervera R, Navarro M, Pallares L, Lopez-Soto A, Vivancos J, et al. Systemic lupus erythematosus in men: clinical and immunological characteristics. Ann Rheum Dis (1992) 51(9):1050-2. doi:10.1136/ard.51. 9.1050

187. Jacobsen S, Petersen J, Ullman S, Junker P, Voss A, Rasmussen JM, et al. A multicentre study of 513 Danish patients with systemic lupus erythematosus. I. Disease manifestations and analyses of clinical subsets. Clin Rheumatol (1998) 17(6):468-77. doi:10.1007/BF01451282

188. Faezi ST, Hosseini Almodarresi M, Akbarian M, Gharibdoost F, Akhlaghi M, Jamshidi A, et al. Clinical and immunological pattern of systemic lupus erythematosus in men in a cohort of 2355 patients. Int J Rheum Dis (2014) 17(4):394-9. doi:10.1111/1756-185X.12268

189. Borba EF, Araujo DB, Bonfa E, Shinjo SK. Clinical and immunological features of 888 Brazilian systemic lupus patients from a monocentric cohort: comparison with other populations. Lupus (2013) 22(7):744-9. doi:10.1177/0961203313490432

190. Molina JF, Drenkard C, Molina J, Cardiel MH, Uribe O, Anaya JM, et al. Systemic lupus erythematosus in males. A study of 107 Latin American patients. Medicine (1996) 75(3):124-30. doi:10.1097/00005792-19960500000002

191. Alonso MD, Martinez-Vazquez F, Riancho-Zarrabeitia L, Diaz de Teran T, Miranda-Filloy JA, Blanco R, et al. Sex differences in patients with systemic lupus erythematosus from Northwest Spain. Rheumatol Int (2014) 34(1):11-24. doi:10.1007/s00296-013-2798-9

192. Koh WH, Fong KY, Boey ML, Feng PH. Systemic lupus erythematosus in 61 Oriental males. A study of clinical and laboratory manifestations. $\mathrm{Br}$ J Rheumatol (1994) 33(4):339-42. doi:10.1093/rheumatology/33.4.339

193. Azizah MR, Ainol SS, Kong NC, Normaznah Y, Rahim MN. Gender differences in the clinical and serological features of systemic lupus erythematosus in Malaysian patients. Med J Malaysia (2001) 56(3):302-7.

194. Renau AI, Isenberg DA. Male versus female lupus: a comparison of ethnicity, clinical features, serology and outcome over a 30 year period. Lupus (2012) 21(10):1041-8. doi:10.1177/0961203312444771

195. Andrade RM, Alarcon GS, Fernandez M, Apte M, Vila LM, Reveille JD. Accelerated damage accrual among men with systemic lupus erythematosus: XLIV. Results from a multiethnic US cohort. Arthritis Rheum (2007) 56(2):622-30. doi:10.1002/art.22375

196. Hwang J, Lee J, Ahn JK, Park E-J, Cha H-S, Koh E-M. Clinical characteristics of male and female Korean patients with systemic lupus erythematosus: a comparative study. Korean J Intern Med (2015) 30(2):242-9. doi:10.3904/ kjim.2015.30.2.242

197. Cooper GS, Parks CG, Treadwell EL, St Clair EW, Gilkeson GS, Cohen PL, et al. Differences by race, sex and age in the clinical and immunologic features of recently diagnosed systemic lupus erythematosus patients in the southeastern United States. Lupus (2002) 11(3):161-7. doi:10.1191/096120 3302 lu161oa

198. Ward MM, Studenski S. Clinical manifestations of systemic lupus erythematosus. Identification of racial and socioeconomic influences. Arch Intern Med (1990) 150(4):849-53. doi:10.1001/archinte.1990.00390160099020

199. Wang YF, Xu YX, Tan Y, Yu F, Zhao MH. Clinicopathological characteristics and outcomes of male lupus nephritis in China. Lupus (2012) 21(13):1472-81. doi:10.1177/0961203312458467

200. Lee W, Reveille JD, Davis JC, Learch TJ, Ward MM, Weisman MH. Are there gender differences in severity of ankylosing spondylitis? Results from the PSOAS cohort. Ann Rheum Dis (2007) 66(5):633-8. doi:10.1136/ard.2006.060293

201. Ortega Castro R, Font Ugalde P, Castro Villegas MC, Calvo Gutierrez J, Munoz Gomariz E, Zarco Montejo P, et al. Different clinical expression of patients with ankylosing spondylitis according to gender in relation to time since onset of disease. Data from REGISPONSER. Reumatol Clin (2013) 9(4):221-5. doi:10.1016/j.reuma.2012.09.008

202. Jung YO, Kim I, Kim S, Suh CH, Park HJ, Park W, et al. Clinical and radiographic features of adult-onset ankylosing spondylitis in Korean patients: comparisons between males and females. J Korean Med Sci (2010) 25(4):532-5. doi:10.3346/jkms.2010.25.4.532

203. Shahlaee A, Mahmoudi M, Nicknam MH, Farhadi E, Fallahi S, Jamshidi AR. Gender differences in Iranian patients with ankylosing spondylitis. Clin Rheumatol (2015) 34(2):285-93. doi:10.1007/s10067-013-2439-4

204. Hebeisen M, Neuenschwander R, Scherer A, Exer P, Weber U, Tamborrini G, et al. Response to tumor necrosis factor inhibition in male and female patients with ankylosing spondylitis: data from a Swiss Cohort. J Rheumatol (2018) 45(4):506-12. doi:10.3899/jrheum.170166 
205. Webers C, Essers I, Ramiro S, Stolwijk C, Landewe R, van der Heijde D, et al. Gender-attributable differences in outcome of ankylosing spondylitis: longterm results from the outcome in Ankylosing Spondylitis International Study. Rheumatology (Oxford) (2016) 55(3):419-28. doi:10.1093/rheumatology/ kev340

206. Roussou E, Sultana S. Spondyloarthritis in women: differences in disease onset, clinical presentation, and bath ankylosing spondylitis disease activity and functional indices (BASDAI and BASFI) between men and women with spondyloarthritides. Clin Rheumatol (2011) 30(1):121-7. doi:10.1007/ s10067-010-1581-5

207. Ibn Yacoub Y, Amine B, Laatiris A, Hajjaj-Hassouni N. Gender and disease features in Moroccan patients with ankylosing spondylitis. Clin Rheumatol (2012) 31(2):293-7. doi:10.1007/s10067-011-1819-x

208. Tournadre A, Pereira B, Lhoste A, Dubost JJ, Ristori JM, Claudepierre P, et al. Differences between women and men with recent-onset axial spondyloarthritis: results from a prospective multicenter French cohort. Arthritis Care Res (Hoboken) (2013) 65(9):1482-9. doi:10.1002/acr.22001

209. de Carvalho HM, Bortoluzzo AB, Goncalves CR, da Silva JA, Ximenes AC, Bertolo $\mathrm{MB}$, et al. Gender characterization in a large series of Brazilian patients with spondyloarthritis. Clin Rheumatol (2012) 31(4):687-95. doi:10.1007/s10067-011-1890-3

210. Landi M, Maldonado-Ficco H, Perez-Alamino R, Maldonado-Cocco JA, Citera G, Arturi P, et al. Gender differences among patients with primary ankylosing spondylitis and spondylitis associated with psoriasis and inflammatory bowel disease in an iberoamerican spondyloarthritis cohort. Medicine (2016) 95(51):e5652. doi:10.1097/MD.0000000000005652

211. Baraliakos X, Listing J, von der Recke A, Braun J. The natural course of radiographic progression in ankylosing spondylitis: differences between genders and appearance of characteristic radiographic features. Curr Rheumatol Rep (2011) 13(5):383-7. doi:10.1007/s11926-011-0192-8

212. A.A.R.D. Association. Autoimmune Disease List. (2017). Available from: https://www.aarda.org/diseaselist/ (accessed June 20, 2017)

213. Hayter SM, Cook MC. Updated assessment of the prevalence, spectrum and case definition of autoimmune disease. Autoimmun Rev (2012) 11(10):75465. doi:10.1016/j.autrev.2012.02.001

214. Arroyo-Ávila M, Santiago-Casas Y, McGwin G, Cantor RS, Petri M, RamseyGoldman R, et al. Clinical associations of anti-Smith antibodies in PROFILE: a multi-ethnic lupus cohort. Clin Rheumatol (2015) 34(7):1217-23. doi:10.1007/s10067-015-2941-y

215. Reveille JD. Predictive value of autoantibodies for activity of systemic lupus erythematosus. Lupus (2004) 13(5):290-7. doi:10.1191/0961203303lu1015oa

216. Vila LM, Molina MJ, Mayor AM, Peredo RA, Santaella ML, Vila S. Clinical and prognostic value of autoantibodies in puerto Ricans with systemic lupus erythematosus. Lupus (2006) 15(12):892-8. doi:10.1177/096120330 6069352

217. Alba P, Bento L, Cuadrado MJ, Karim Y, Tungekar MF, Abbs I, et al. Anti-dsDNA, anti-Sm antibodies, and the lupus anticoagulant: significant factors associated with lupus nephritis. Ann Rheum Dis (2003) 62(6):556-60. doi:10.1136/ard.62.6.556

218. Janwityanuchit S, Verasertniyom O, Vanichapuntu M, Vatanasuk M. Anti-Sm: its predictive value in systemic lupus erythematosus. Clin Rheumatol (1993) 12(3):350-3. doi:10.1007/BF02231577

219. Martinez-Cordero E, Martinez-Miranda E, Negrete-Garcia MC, Padilla A, Aguilar Leon DE. Anti-dsDNA and Sm autoantibodies in systemic lupus erythematosus. Clin Rheumatol (1992) 11(3):341-5. doi:10.1007/ BF02207190
220. Mond CB, Peterson MGE, Rothfield NF. Correlation of anti-Ro antibody with photosensitivity rash in systemic lupus erythematosus patients. Arthritis Rheum (1989) 32(2):202-4. doi:10.1002/anr.1780320213

221. McCauliffe D. Cutaneous diseases in adults associated with anti-Ro/ SS-A autoantibody production. Lupus (1997) 6(2):158-66. doi:10.1177/ 096120339700600211

222. Fukuda MV, Lo SC, de Almeida CS, Shinjo SK. Anti-Ro antibody and cutaneous vasculitis in systemic lupus erythematosus. Clin Rheumatol (2008) 28(3):301. doi:10.1007/s10067-008-1043-5

223. Alexander EL, Arnett FC, Provost TT, Stevens M. Sjögren's syndrome: association of anti-ro(ss-a) antibodies with vasculitis, hematologic abnormalities, and serologic hyperreactivity. Ann Intern Med (1983) 98(2):155-9. doi:10.7326/0003-4819-98-2-155

224. Kurien BT, Newland J, Paczkowski C, Moore KL, Scofield RH. Association of neutropenia in systemic lupus erythematosus (SLE) with anti-Ro and binding of an immunologically cross-reactive neutrophil membrane antigen. Clin Exp Immunol (2000) 120(1):209-17. doi:10.1046/j.1365-2249.2000.01195.x

225. Maddison P, Mogavero H, Provost TT, Reichlin M. The clinical significance of autoantibodies to a soluble cytoplasmic antigen in systemic lupus erythematosus and other connective tissue diseases. J Rheumatol (1979) 6(2):189-95.

226. Chung JK, Kim MK, Wee WR. Prognostic factors for the clinical severity of keratoconjunctivitis sicca in patients with Sjögren's syndrome. $\mathrm{Br}$ J Ophthalmol (2012) 96(2):240-5. doi:10.1136/bjo.2011.202812

227. Harley JB, Alexander EL, Bias WB, Fox OF, Provost TT, Reichlin M, et al. Anti-Ro (SS-A) and Anti-La (SS-B) in patients with Sjögren's syndrome. Arthritis Rheum (1986) 29(2):196-206. doi:10.1002/art.1780290207

228. Franceschini F, Cretti L, Quinzanini M, Lodi Rizzini F, Cattaneo R. Deforming arthropathy of the hands in systemic lupus erythematosus is associated with antibodies to SSA/Ro and to SSB/La. Lupus (1994) 3(5):419-22. doi:10.1177/096120339400300510

229. Simmons-O'Brien E, Chen S, Watson R, Antoni C, Petri M, Hochberg M, et al. One hundred anti-Ro (SS-A) antibody positive patients: a 10-year follow-up. Medicine (1995) 74(3):109-30. doi:10.1097/00005792-199505000-00001

230. Doria A, Zen M, Canova M, Bettio S, Bassi N, Nalotto L, et al. SLE diagnosis and treatment: when early is early. Autoimmun Rev (2010) 10(1):55-60. doi:10.1016/j.autrev.2010.08.014

231. Hennes EM, Zeniya M, Czaja AJ, Parés A, Dalekos GN, Krawitt EL, et al. Simplified criteria for the diagnosis of autoimmune hepatitis. Hepatology (2008) 48(1):169-76. doi:10.1002/hep.22322

232. Kerlikowske K, Grady D, Rubin SM, Sandrock C, Ernster VL. Efficacy of screening mammography. A meta-analysis. JAMA (1995) 273(2):149-54. doi:10.1001/jama.1995.03520260071035

Conflict of Interest Statement: The authors declare that the research was conducted in the absence of any commercial or financial relationships that could be construed as a potential conflict of interest.

The reviewer YL and handling Editor declared their shared affiliation.

Copyright (C) 2018 Purnamawati, Ong, Deshpande, Tan, Masurkar, Low and Drum. This is an open-access article distributed under the terms of the Creative Commons Attribution License (CC BY). The use, distribution or reproduction in other forums is permitted, provided the original author(s) and the copyright owner are credited and that the original publication in this journal is cited, in accordance with accepted academic practice. No use, distribution or reproduction is permitted which does not comply with these terms. 